\title{
The possible effect of coenzyme Q10 and captopril on acetaminophen-induced encephalopathy in rats: possible influence on autophagy, antioxidant and $\mathrm{Na}^{+} / \mathrm{K}^{+}$ATPase
}

\author{
Heba M. Galal ${ }^{1,2}$, Manal M. Sayed ${ }^{3}$, Eman S. H. Abd Allah ${ }^{1}$
} ${ }^{1}$ Medical Physiology Department, Faculty of Medicine, Assiut University, Assiut, Egypt
${ }^{2}$ Medical Physiology Department, Faculty of Medicine, Jouf University, Sakaka,
Saudi Arabia
${ }^{3}$ Histology and Cell Biology Department, Faculty of Medicine, Assiut University, Assiut,
Egypt

Submitted: 10 January 2020

Accepted: 17 April 2020

Arch Med Sci

DOI: https://doi.org/10.5114/aoms.2020.100591

Copyright $\odot 2020$ Termedia \& Banach

\section{Abstract}

Introduction: Induction of autophagy could protect against acetaminophen (APAP)-induced hepatotoxicity; however, little is known about the role of autophagy in APAP-induced encephalopathy (APAP-E). This study aimed to evaluate the effects of coenzyme Q10 (CoQ10) and captopril on APAP-E. Material and methods: Forty-eight rats were randomly allotted to 4 equal groups: control, an APAP-E, coenzyme Q10-treated (CoQ10-treated), and captopril-treated groups. Behavioral tests were conducted. Serum ammonia and total antioxidant capacity (TAC) and hippocampal $\mathrm{Na}^{+} / \mathrm{K}^{+}$ATPase activity were measured. The expression levels of hippocampal microtubule-associated protein light chain 3 (LC3-II) and beclin-1 mRNA were detected using quantitative polymerase chain reaction ( $q P C R$ ). General histological, immunohistochemical staining for glial fibrillary acid protein (GFAP) and electron microscopy (EM) of the hippocampus were performed.

Results: In the APAP-E group, serum ammonia was increased significantly, hippocampal LC3-II and beclin-1 mRNA were elevated insignificantly, while serum TAC and the activity of hippocampal $\mathrm{Na}^{+} / \mathrm{K}^{+}$ATPase were reduced significantly compared with the control group. APAP-E rats showed remarkable degenerative changes in CA1 pyramidal neurons in the form of electron-dense cytoplasm with ill-defined nuclei and accumulation of lysosomal structure-like dense bodies. Increased immunoreactivity of astrocytes for GFAP was observed. Treatment with either CoQ10 or captopril significantly reduced ammonia levels, increased hippocampal LC3-II and beclin-1 mRNA, increased serum TAC and $\mathrm{Na}^{+} / \mathrm{K}^{+}$ATPase activity, and noticeably ameliorated the hippocampal neuronal changes. EM revealed restoration of the normal structure of pyramidal neurons. These effects were more obvious in CoQ10-treated than captopril-treated rats.

Conclusions: CoQ10 and captopril have neuroprotective effects on APAP-E via enhancing LC3-II, beclin-1 mRNA expression, serum TAC level and hippocampal $\mathrm{Na}^{+} / \mathrm{K}^{+}$ATPase activity.

Key words: acetaminophen-induced encephalopathy, beclin-1, coenzyme Q10, captopril, hippocampus, microtubule-associated protein light chain-3, $\mathrm{Na}^{+} / \mathrm{K}^{+}$ATPase.

\author{
Corresponding author: \\ Dr. Heba M. Galal \\ Medical Physiology \\ Department \\ Faculty of Medicine \\ Assiut University \\ Assiut, Egypt \\ Phone: 01000788437 \\ E-mail: Hebamgalal17@aun. \\ edu.eg
}




\section{Introduction}

Acetaminophen-induced encephalopathy (APAP-E) is a consequence of ingestion of high doses of acetaminophen. Increased ammonia, glutamate, calcium influx, mitochondrial damage and oxidative stress are suggested to play a role [1]. Acetaminophen (APAP) also increases both the activity and the protein levels of neuronal cytochrome P450 $2 \mathrm{E} 1$, in which disturbed mitochondrial functions lead in turn to neuronal death [2]. The pathological effects of paracetamol on the liver and kidneys have been studied extensively, but little information is available about its toxic effects on nervous tissue [3].

Autophagy is a lysosomal intracellular recycling process of bulk proteins, unfolded proteins, and aged organelles through the formation of autophagosomes. Under physiologic conditions, it is a tightly regulated process that preserves energy resources, removes unnecessary elements, and ameliorates stressful conditions such as oxidative stress and endoplasmic reticulum stress, thus maintaining the vitality of cells [4]. Mitochondrial dysfunction together with concomitant formation of reactive oxygen species (ROS) has been shown to mediate autophagic cell death [5]. Boosted autophagy has been detected in animal models of stroke, excitotoxicity, traumatic brain injury, and in patients with Alzheimer's disease and critical illness [6]. While autophagy is primarily a cell protection mechanism, its exacerbation and/or failure can also play a role in cell death [7]. APAP modulates liver autophagy [8] and induction of autophagy could protect against APAP-induced hepatotoxicity.

Coenzyme Q10 (CoQ10) is a fat-soluble quinone antioxidant, has a structural similarity to vitamins $\mathrm{K}$ and $\mathrm{E}$, is manufactured by body cells and is present in abundant amounts in the human diet [9]. It serves as a cofactor in the mitochondrial respiratory chain, in addition to being a normal scavenger of free radicals, preventing the irreversible cycle of peroxidation to cellular lipids, proteins and DNA. CoQ10 supplementation may provide favorable outcomes via improving and preventing pathological illness such as neurodegenerative diseases, cancer, metabolic syndrome, hypertension, insulin-resistant diabetes mellitus, liver ailments [10], and statin-induced myopathy [11]. These outcomes could be attributed to the reduction of inflammatory cytokine (IL-6) [12] or due to maintaining the mitochondrial function [13]. Several studies have proved the involvement of the brain renin-angiotensin system in memory dysfunction [14]. Recently, CoQ10 was found to ameliorate cardiac damage in mice nourished on a diet with high fat content via upregulation of autophagy-related gene (LC3-II) besides its antioxidant and anti-inflammatory effects [15]. It was reported that
CoQ10 by its antioxidant, anti-inflammatory, and anti-apoptotic effects can have a therapeutic role in APAP-induced hepatotoxicity as well as metabolic stress-induced liver damage [16]. Increased generation of angiotensin II (Ang II) in neuronal tissues induces inflammatory responses and oxidative stress in the brain, impairing learning and memory [17]. Captopril is the first orally active angiotensin converting enzyme (ACE) inhibitor that markedly reduced brain damage in various neurodegenerative diseases via its antioxidant effects [18].

The role of autophagy in APAP-E and the effects of both CoQ10 and captopril on it are still uncertain. Thus, the present experimental study was performed to evaluate the effects of oral administration of CoQ10 or captopril for 7 days, as antioxidant autophagy inducers, on an acetaminophen-induced encephalopathy rat model.

\section{Materials and methods}

\section{Drugs and treatment}

APAP (Sigma-Aldrich, St. Louis, MO, USA) was prepared as $20 \%$ suspension in saline $(0.9 \%)$ stabilized by $0.2 \%$ gum with continuous magnetic stirring and injected intraperitoneally (IP) at a dose of $800 \mathrm{mg} / \mathrm{kg} /$ day for 3 days to in duce encephalopathy [19]. CoQ10 and captopril (Sigma-Aldrich Chemical Company, Cairo, Egypt) were dissolved in $0.9 \%$ saline and given orally for 7 days at a dose of $50 \mathrm{mg} / \mathrm{kg} /$ day according to Abd Allah and Gomaa [20] and Ashkani Esfahani et al. [21], respectively.

\section{Animals}

Adult male rats of Wistar Albino strain, weighing 125-150 g were used in this study. Rats were accommodated in clean, well-ventilated cages (up to 4 rats per cage) in the animal house of the Faculty of Medicine, Assiut University, from which rats were purchased. Animals were kept in a room with an average temperature of $22 \pm 2^{\circ} \mathrm{C}$, under a 12 hours light-dark rhythm with free access to basal diets and water throughout the study period. They were adapted for a week prior the commencement of the experimental procedures. The Ethics Committee at the Faculty of Medicine, Assiut University, Egypt approved the research proposal. The authors followed both Guidelines of Experiments on Animals and the Guide for the Care and Use of Laboratory Animals during all experimental procedures [22].

\section{Experimental design}

Forty-eight Wistar Albino rats were used for this study. Rats were randomly allocated to 4groups, twelve animals each, as follows: 
- Control group: animals received the vehicle ( $0.9 \%$ saline and $0.2 \%$ gum) IP and orally, respectively.

- APAP-induced encephalopathy (APAP-E) group: animals received IP injections of APAP at a dose of $800 \mathrm{mg} / \mathrm{kg} /$ day for 3 days [19] and were allowed to survive for a week after the last given dose.

- CoQ10-treated group animals received IP injections APAP for 3 days [19] then oral CoQ10 for 7 days beginning on the $4^{\text {th }}$ day [21].

- Captopril-treated group animals received IP injections of APAP for 3 days then oral captopril for 7 days beginning on the $4^{\text {th }}$ day [20].

\section{Behavioral tests}

Behavioral tests were performed at the end of the $7^{\text {th }}$ treatment day.

\section{Novel object recognition test}

Novel object recognition test (NOR) is a sensitive, accurate and trustworthy memory assessment tool [23]. This test depends on the fact that the normal behavior of rodents is to spend more time exploring a novel object when exposed to both novel and familiar objects at the same time. The habituation, familiarization and test phases are the components of the NOR test. Briefly, at the beginning of the test, each rat was allowed to become accustomed to the empty, open, stainless steel-made box for $3 \mathrm{~min}$. Then, the rat was taken out of the open box and positioned in its cage. Meanwhile, 2 identical sample objects (familiar red round objects) were placed in the open box. Thereafter, each rat was permitted to freely explore (familiarization) the 2 identical items for $3 \mathrm{~min}$; the rat was put in the center of the open box with its back to the items. Afterwards, the test phase was carried out using 2 objects, a red round object (familiar) and a yellow pyramid (novel) after four-hour (intermediate memory) and twenty-fourhour (long-term memory) retention periods. Each rat was allowed to explore the familiar and the novel objects for 3 min during the test phase.

The discrimination index (DI) was calculated using the following equation: $(\mathrm{DI}=(\mathrm{TN}-\mathrm{TF}) /(\mathrm{TN}$ $+\mathrm{TF})$ ), where TN is the exploration time for the novel object and TF is the exploration time for the familiar object [24].

\section{Activity cage}

An activity cage (UGO Basile; model 7401, Camerio, VA, Italy) was used to assess the spontaneous locomotor activity. Rats were located separately in the activity cage chamber for $5 \mathrm{~min}$. The spontaneous locomotor activity was counted and expressed in terms of counts per $5 \mathrm{~min}$; habituation for $3 \mathrm{~min}$ was done before each testing session [25].

\section{Biochemical examinations}

\section{Blood}

Immediately before the euthanasia, blood samples from fasted rats were collected from each rat via the retro-orbital vein and centrifuged for $15 \mathrm{~min}$ at $1008 \mathrm{~g}$. The transparent sera were taken up and kept until use at $-20^{\circ} \mathrm{C}$.

\section{Determination of serum ammonia}

A commercially available colorimetric kit obtained from Bio-Diagnostics (Giza, Egypt) was used to estimate serum ammonia level. Briefly, freshly obtained serum was transferred immediately to trichloroacetic acid, centrifuged, and the ammonia concentration was determined in the supernatant using the Berthelot reaction. The blue indophenol dye generated in the reaction is proportional to the ammonia concentration.

\section{Determination of serum total antioxidant capacity}

Colorimetric measurement of serum total antioxidant capacity (TAC) was done using the commercially available kit (Bio-Diagnostics, Giza, Egypt) as described by Koracevic et al. [26]. Briefly, serum antioxidants eliminated a definite amount of exogenously provided hydrogen peroxide $\left(\mathrm{H}_{2} \mathrm{O}_{2}\right)$ and the remaining $\mathrm{H}_{2} \mathrm{O}_{2}$ was determined using an enzymatic reaction that utilizes the conversion of 3,5-dichloro-2-hydroxy benzenesulfonate to a colored product.

\section{Tissue}

The rats (6 rats from each group) were anesthetized with a piece of cotton soaked with $10 \mathrm{ml}$ of halothane. Then, rats were sacrificed by decapitation. One hippocampus was taken from each rat, and was snap frozen in liquid nitrogen, then was stored at $-80^{\circ} \mathrm{C}$ until further use for quantitative polymerase chain reaction (qPCR). The other hippocampus was homogenized in ice-cold phosphate-buffered saline $(\mathrm{pH} 7.4)$ by Glas-Col Homogenizer AQ5 and centrifuged at $10000 \mathrm{rpm}$ for $10 \mathrm{~min}$ by ultra-centrifugation (Hettich EBA 12) at about $4^{\circ} \mathrm{C}$ and the supernatants were kept at $-20^{\circ} \mathrm{C}$ until used.

\section{Determination of hippocampal $\mathrm{Na}^{+} / \mathrm{K}^{+}$ ATPase activity}

Hippocampal $\mathrm{Na}^{+} / \mathrm{K}^{+}$ATPase activity was calculated using the spectrophotometric chemical method defined by Sehirli et al. [27]. Determination of $\mathrm{Na}^{+} / \mathrm{K}^{+}$ATPase activity was based on measurement of the quantity of Pi produced during incubation of hippocampal homogenate $(100 \mu \mathrm{l})$ with $900 \mu \mathrm{l}$ of mixture solution produced by 
adding $\mathrm{Na}_{2}$ ATP in a concentration of $3 \mathrm{mmol} / \mathrm{l}$ to a pre-incubated medium mixture containing $\mathrm{NaCl}$, $\mathrm{KCl}, \mathrm{MgCL}_{2}$, ethylenediaminetetraacetic acid, and Tris $\mathrm{HCl}(\mathrm{PH} 7.4)$ at the following molar concentrations $100,5,6,0.1,30 \mathrm{mmol} / \mathrm{l}$, respectively, at $37^{\circ} \mathrm{C}$ for $30 \mathrm{~min}$. Thereafter, the reaction was ended by putting the incubated mixtures on ice. Lastly, molybdate reagent was added and the amount of inorganic phosphate (Pi) formed was calculated using a spectrophotometer (Shimadzu, Kyoto, Japan) at the wavelength $390 \mathrm{~nm}$ according to Atkinson et al. [28]. Hippocampal $\mathrm{Na}^{+} / \mathrm{K}^{+}$ATPase activity was expressed as $\mathrm{nmol} \mathrm{Pi} / \mathrm{mg}$ protein/h. The protein concentration of the homogenate supernatant was measured as described by Lowry et al. [29].

\section{Quantitative polymerase chain reaction}

RNA (100 ng), extracted from the hippocampus via TRIzol reagent, was reverse transcribed using a high capacity cDNA reverse transcription kit (Applied Biosystems, USA) following the manufacturer's instructions. qPCR was carried out using a QuantiTect SYBR Green PCR Kit (Qiagen, USA) and Mx3005P qPCR detection system (Agilent Technologies, USA). The PCR protocol comprised $5 \mathrm{~min}$ at $95^{\circ} \mathrm{C} ; 40$ cycles of denaturation $\left(30 \mathrm{~s}\right.$ at $\left.95^{\circ} \mathrm{C}\right)$; annealing $\left(30 \mathrm{~s}\right.$ at $60^{\circ} \mathrm{C}$ for all genes); and polymerization at $72^{\circ} \mathrm{C}$ for $1 \mathrm{~min}$. To confirm the product purity, a melting dissociation curve was generated by heating to $95^{\circ} \mathrm{C}$ for $15 \mathrm{~s}, 60^{\circ} \mathrm{C}$ for $15 \mathrm{~s}$, and $95^{\circ} \mathrm{C}$ for another 15 s. Each sample was run in duplicate. The primer sequences were as follows: LC3-II (forward, 5'-CATGGGCACAGATGAAGACAC-3'), reverse 5'-GCCAGATGTTCATCCACTTTC, Beclin-1 (forward, 5'-ACCAGGAGGAAGCTCAGTACC-3', reverse 5'-CAGGCAGCATTGATTTCATTC) [30], and gene vs. a housekeeping reference gene (GAPDH; forward, 5'-ATGGGAGTTGCTGTTGAAGTCA-3', reverse 5'-CCGAGGGCCCACTAAAGG-3') [31]. The relative expression level of the LC3-II and beclin-1 genes vs. GAPDH were calculated using the 2- $\Delta \Delta \mathrm{Ct}$ method as described by Navarro et al. [32] and were represented as the fold change relative to the control group; its value is 100 .

\section{Histological study}

At the end of the experiment, 6 rats from each group were anesthetized by an IP injection of thiopental sodium (50 mg/kg) [33]. The heart of an anesthetized rat was perfused with saline $(0.9 \%)$ until it cleared from blood. Finally, perfusion with $10 \%$ neutral-buffered formalin $(\mathrm{pH} 7.2)$ in 3 rats and with $4 \%$ glutaraldehyde in the other 3 rats from each group was done. Afterwards, the skull was opened and the brain was carefully separated from the skull and was immersed in the appropriate fixative.

\section{Light microscopy}

The brain samples were fixed in 10\% neutral buffered formalin, dehydrated, washed, and coated in paraffin. 4-6 $\mu \mathrm{m}$ coronal paraffin sections were cut and stained with hematoxylin and eosin (H\&E) for general histological study [34] and glial fibrillary acid protein (GFAP) antibodies for immunohistochemical detection of astrocytes [35].

\section{Immunohistochemical staining}

Paraffin sections were immunostained with a mouse anti-rat GFAP monoclonal antibody (GA-5; Thermo Fisher Scientific Co, Fremont, California, USA) using the modified avidin-biotin-peroxidase technique. For color development, a universal kit, avidin-biotin-peroxidase system (Thermo Fisher Scientific Co, Fermont, California, USA) was used.

Briefly, paraffin sections were placed on positive-charged glass slides and passed through complete deparaffinization in xylene and rehydration in descending grades of ethanol. The sections were incubated with $10 \% \mathrm{H}_{2} \mathrm{O}_{2}$ for 10 min to block endogenous peroxidase activity. To unmask the antigenic sites, another incubation of the sections for $10 \mathrm{~min}$ was done with $0.01 \mathrm{~mol} / \mathrm{L}$ citrate buffer ( $\mathrm{pH}$ 6.0) and then boiled in a microwave oven for $5 \mathrm{~min}$. After that, incubation with the primary (anti-GFAP, 1:100) antibody was done for 1 hour and then washed and incubated with the secondary biotinylated antibody and then with the avidin-biotin complex. Lastly, the specimens were formed with $0.05 \%$ diaminobenzidine and counterstained with hematoxylin, dehydrated, cleared and mounted. Glial fibrillary acid protein-containing cells (astrocytes) appear brown and their nuclei appear blue [36].

\section{Transmission electron microscopy}

The hippocampi were dissected out of the brains, immersed in a cacodylate buffer ( $\mathrm{pH} 7.4)$ containing $4 \%$ glutaraldehyde for 24 hours and after that fixed in $1 \%$ osmium tetroxide in a phosphate buffer for 2 hours. The specimens were rinsed in the same solution buffer, alcohol dehydrated; propylene oxide cleared and in turn Epon-812 substitute was inserted in place. Semithin sections $(0.5-1 \mu \mathrm{m})$ were then cut with glass knives on the ultratome and stained with $1 \%$ toluidine blue $(\mathrm{pH} 7.3)$ for light microscope inspection [37]. Ultrathin specimens $(50-80 \mathrm{~nm})$ from the CA1 area were sliced, placed on copper grids, on a Reichert ultratome, and were contrasted with uranyl acetate and lead citrate. These parts were analyzed using the transmission electron micros- 
copy (Jeol EM-100 CX11; Japanese optic electron lab, Tokyo, Japan) and snapped at the Assiut University Electron Microscope Unit at $80 \mathrm{kV}$.

\section{Morphometric study}

Five non-overlapping fields in 3 consecutive sections from 3 different rats were used for morphometric study. In toluidine blue-stained sections, the thickness of the pyramidal cell layer and the number of dark cells in pyramidal neurons in the CA1 field were determined using $100 \times$ lens. In addition, the number of astrocytes in GFAP-stained sections in the CA1 field was counted using the $100 \times$ lens. All morphometric studies were carried out using image J, which is a program for the processing of open source images based on Java.

\section{Statistical analyses}

Data are presented as the mean \pm standard error of the mean. Kruskal-Wallis $\mathrm{H}$ test followed by the Mann-Whitney $U$ test was applied to assess the statistical differences between groups. A value of $p \leq 0.05$ was considered statistically significant. The association between measured parameters was determined using Spearman's rank correlations in accordance with Knapp and Miller [38]. SPSS software version 20 (SPSS Inc., Chicago, IL, USA) was the statistical program used for doing all statistical analyses of the results.

A

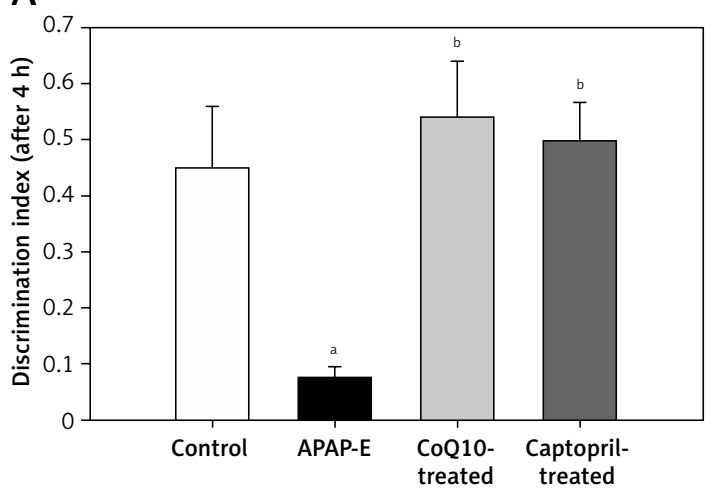

C

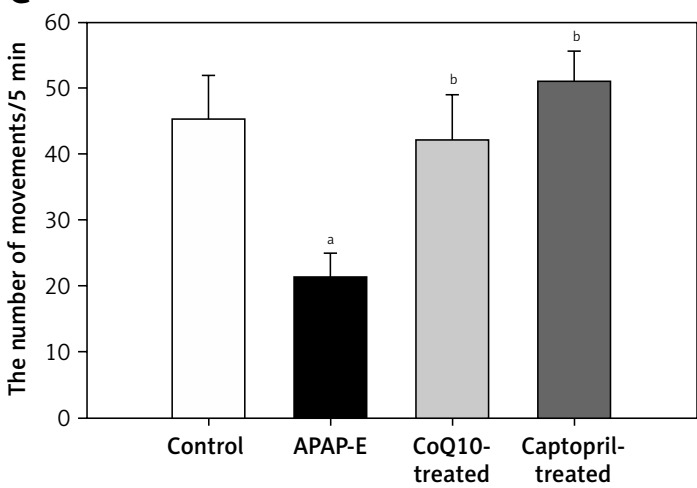

\section{Results}

\section{Behavioral tests}

\section{Novel object recognition test}

There was a marked reduction in DI after 4 and 24 $\mathrm{h}$ of the familiarization phase in APAP-E compared to the control; rats spent equal time exploring novel and old objects or spent a shorter time in exploring a novel object than the familiar object. Treatment with either CoQ10 or captopril resulted in a significant increase in DI after 4 and $24 \mathrm{~h}$ (Figures $1 \mathrm{~A}, \mathrm{~B}$ ).

\section{Activity cage}

A significant decrease in the locomotor activity was noted in rats given APAP compared to the control, CoQ10-treated, and captopril-treated groups ( $p=0.009, p=0.041, p=0.004$; respectively), supporting the occurrence of hepatic encephalopathy. Administration of $\mathrm{CoQ} 10$ or captopril restored the locomotor activity of diseased animals with no significant differences from the control group (Figure $1 \mathrm{C}$ ).

\section{Serum ammonia and total antioxidant capacity}

APAP-E resulted in significantly higher serum ammonia levels compared with levels in the control group (Figure 2 A). In contrast, APAP-E group had a significantly lower serum TAC compared to the control (Figure 2 B). Administration of either CoQ10

B

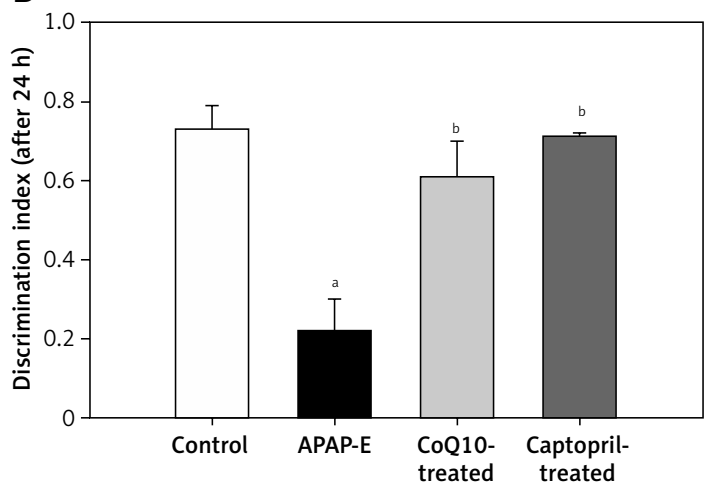

Figure 1. Discrimination index (DI) of novel object recognition test after $4 \mathrm{~h}(\mathrm{~A}), 24 \mathrm{~h}$ (B) of training, and motor activity of rats in activity cage (C) of the different studied groups. DI was calculated as the difference in exploration time for novel and familiar objects divided by the total time of exploration of the novel and familiar objects (DI $=(\mathrm{TN}-\mathrm{TF}) /(\mathrm{TN}$ $+T F)$ ). Data are mean \pm standard error of the mean. aStatistically significant as compared to the control group. bStatistically significant as compared to the APAP-E group. Motor activity was calculated as the number of movements per $5 \mathrm{~min}$ 
A

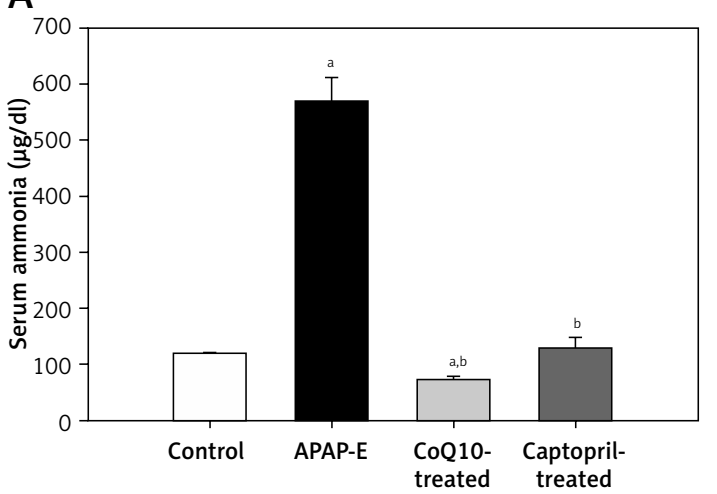

C

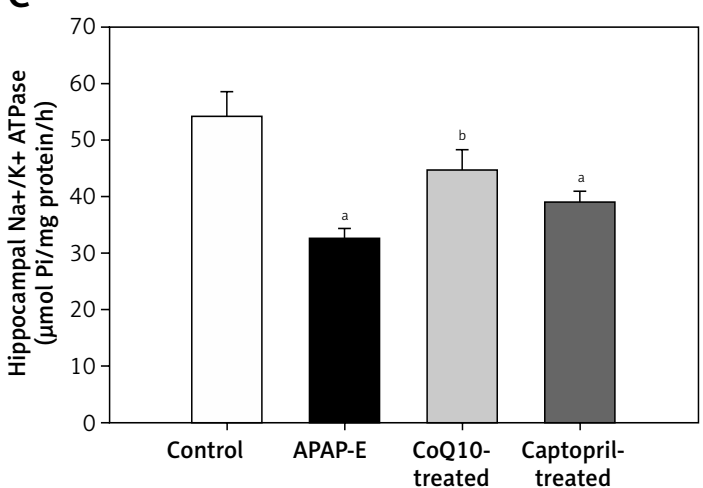

A

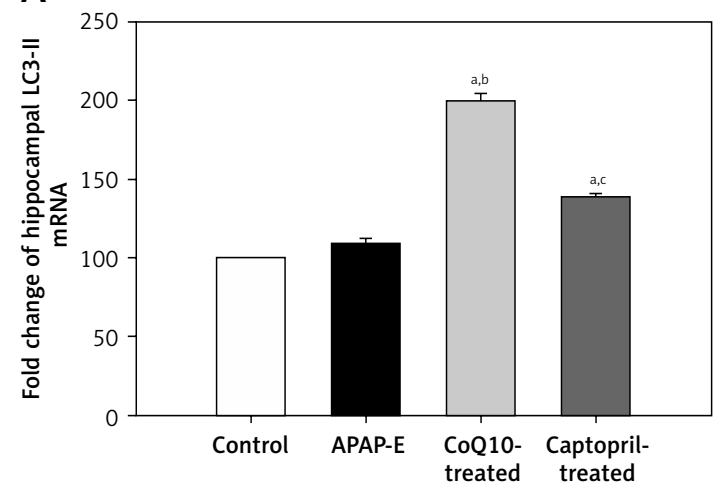

B

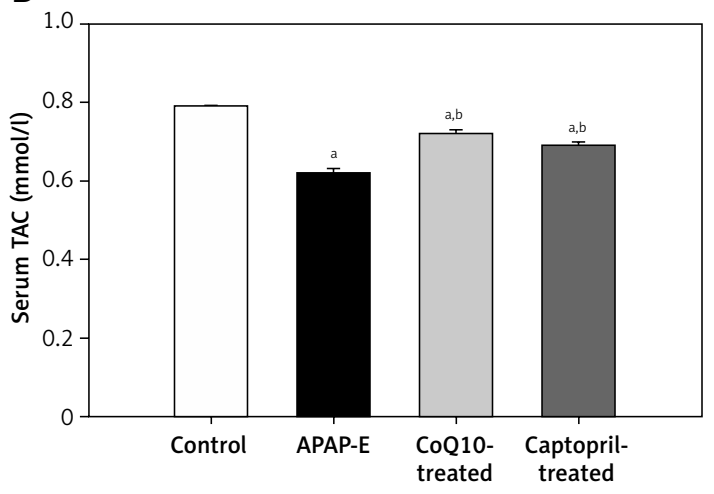

Figure 2. A - Serum ammonia ( $\mu \mathrm{g} / \mathrm{dl}$; A). B - Serum total antioxidant capacity $(\mathrm{mmol} / \mathrm{l})$. B, C - Hippocampal $\mathrm{Na}^{+} / \mathrm{K}^{+}$ATPase activity $(\mu \mathrm{mol} \mathrm{Pi} / \mathrm{mg}$ protein/h) in all groups studied. Data are presented as mean \pm standard error of the mean $(n=6$ in each group). aSignificant as compared to the control group $p<0.05$. 'Significant as compared to the APAP-E group $p<0.05$. The Kruskal-Wallis $\mathrm{H}$ test followed by the Mann-Whitney $U$ test was used for statistical analysis

TAC - total antioxidant capacity.

B

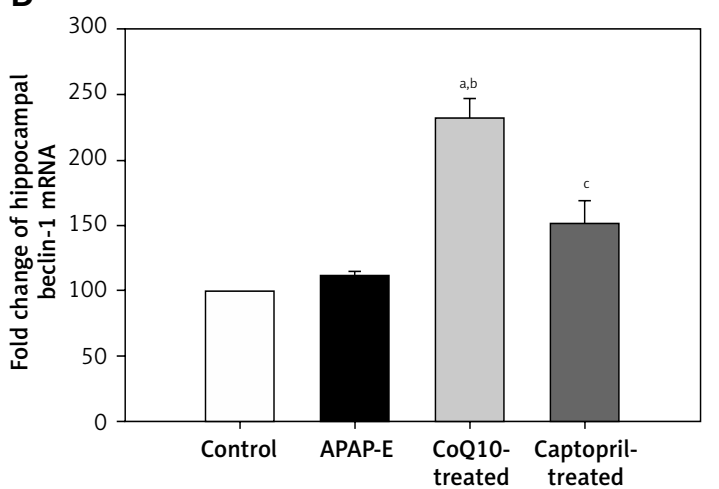

Figure 3. The fold expression of the hippocampal light chain 3 (LC3-II) and beclin-1 mRNA in the different experimental groups. Mean \pm standard error of the mean $(n=6)$ is shown. The relative expression of the LC3-II and beclin-1 gene vs. a housekeeping reference gene was calculated using the equation of 2- $\Delta \Delta \mathrm{Ct}$ and represented as fold change relative to the control group. Autophagy protects against acetaminophen induced encephalopathy

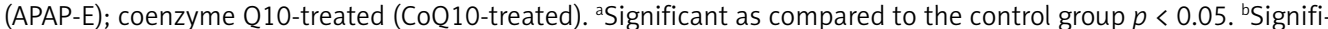
cant as compared to the APAP-E group $p<0.05$. 'Significant as compared to the CoQ10-treated group $p<0.05$. The Kruskal-Wallis $H$ test followed by the Mann-Whitney $U$ test was used for statistical analysis

or captopril resulted in a significant reduction in the serum ammonia and a significant increase in serum TAC levels; however, these changes tended to be more obvious in the CoQ10-treated group compared with captopril-treated group (Figures $2 \mathrm{~A}, \mathrm{~B}$ ).

\section{Hippocampal $\mathrm{Na}^{+} / \mathrm{K}^{+}$ATPase activity}

Hippocampal $\mathrm{Na}^{+} / \mathrm{K}^{+}$ATPase activity was significantly reduced in the APAP-E group as compared with that in the control. CoQ10 and captopril ame- liorated the reduction in $\mathrm{Na}^{+} / \mathrm{K}^{+}$ATPase activity induced by APAP; however, the restoration of $\mathrm{Na}^{+} / \mathrm{K}^{+}$ ATPase activity tended to be more pronounced in the CoQ10-treated group compared with the captopril-treated group (Figure $2 \mathrm{C}$ ).

\section{Expression of hippocampal light chain 3 and beclin-1 mRNA}

Quantitative polymerase chain reaction showed that the fold expression levels of hippocampal 
LC3-II and beclin-1 mRNA were insignificantly higher in the APAP-E group compared with the control group. A further significant increase in the expression of LC3-II and beclin-1 mRNA was observed after administration of CoQ10 or captopril; however, this increase was more pronounced after CoQ10 intake (Figures $3 \mathrm{~A}, \mathrm{~B}$ ).

Spearman's correlation was carried out individually in each group (control, APAP-E, CoQ10-treated, and captopril-treated). Serum ammonia had significant negative correlations with hippocampal $\mathrm{Na}^{+} / \mathrm{K}^{+}$ATPase activity and serum TAC in all groups and no correlation with autophagy markers (LC3-II and beclin-1) in control and APAP-E groups; however, it showed significant negative correlations in CoQ10-treated and captopril-treated groups. Hippocampal $\mathrm{Na}^{+} / \mathrm{K}^{+}$ATPase activity correlated positively with serum TAC in all groups. Significant positive correlations were observed between markers of autophagy and serum TAC and hippocampal $\mathrm{Na}^{+} / \mathrm{K}^{+}$ATPase activity in CoQ10-treated and captopril-treated groups (Table 1 ).

\section{Histological results}

In H\&E-stained section, the hippocampus of the control group showed the U-shaped dentate gyrus, Ammon's horn (AH) or cornu ammonis (CA) with its subdivisions CA1, CA2, CA3 and CA4 fields (Figure $4 \mathrm{~A}$ inset). The semithin section stained with toluidine blue stain of the control group showed that the CA1 field was formed of stratum oriens, stratum pyramidal, and stratum radiatum (SR) (Figure $4 \mathrm{~A}$ ). Pyramidal neurons with large rounded vesicular nuclei, prominent nucleoli, and a considerable amount of basophilic granular cytoplasm were closely packed in the stratum pyramidale (SP) with their long prominent dendrites projecting into the SR. Scattered astrocytes were present in the CA1 field of the control hippocampus. They exhibited pale oval or rounded nuclei surrounded by a thin rim of lightly stained cytoplasm (Figure $4 \mathrm{~A}$ ).

The CA1 field of the hippocampus of the APAP-E group stained with toluidine blue stain showed many scattered pyramidal neurons, which appeared shrunken with irregular darkly stained cytoplasm, pyknotic nuclei, and long dark dendrites. Other neurons appeared normal with large vesicular pale nuclei. In addition, many astrocytes with wide astrocytic processes were seen (Figure 4 B).

The CA1 field of the hippocampus of CoQ10treated rats demonstrated that most of the pyramidal neurons in the SP appeared normal with large vesicular nuclei, prominent nucleoli and long dendrites. Astrocytes with dilated processes around the blood capillary appeared (Figure 4 C). In the captopril-treated group, most of the pyramidal neurons in the SP in the CA1 field appeared normal with

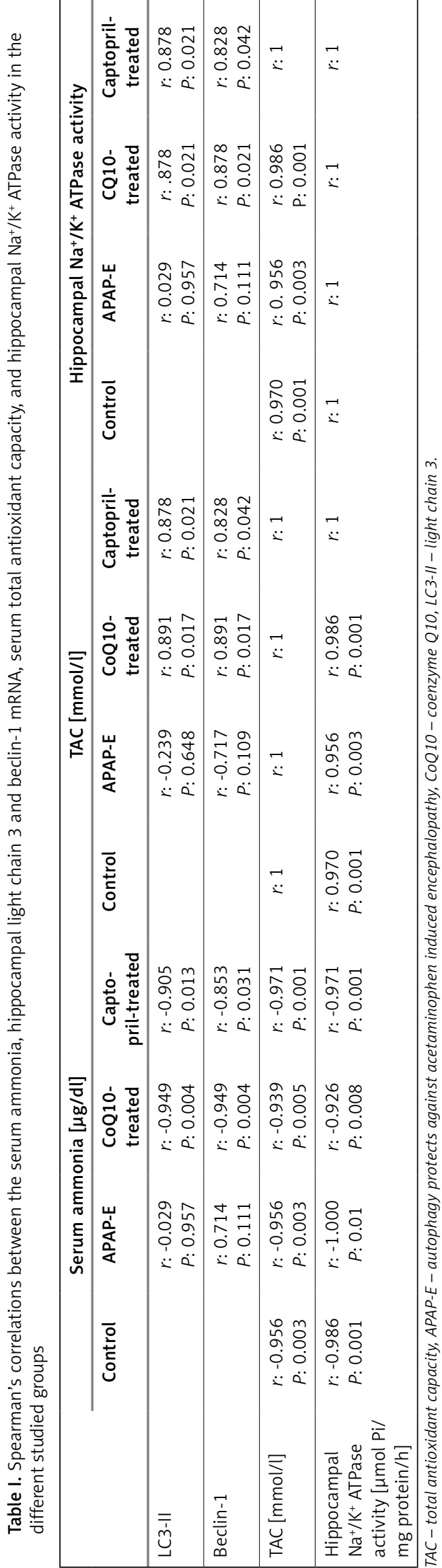



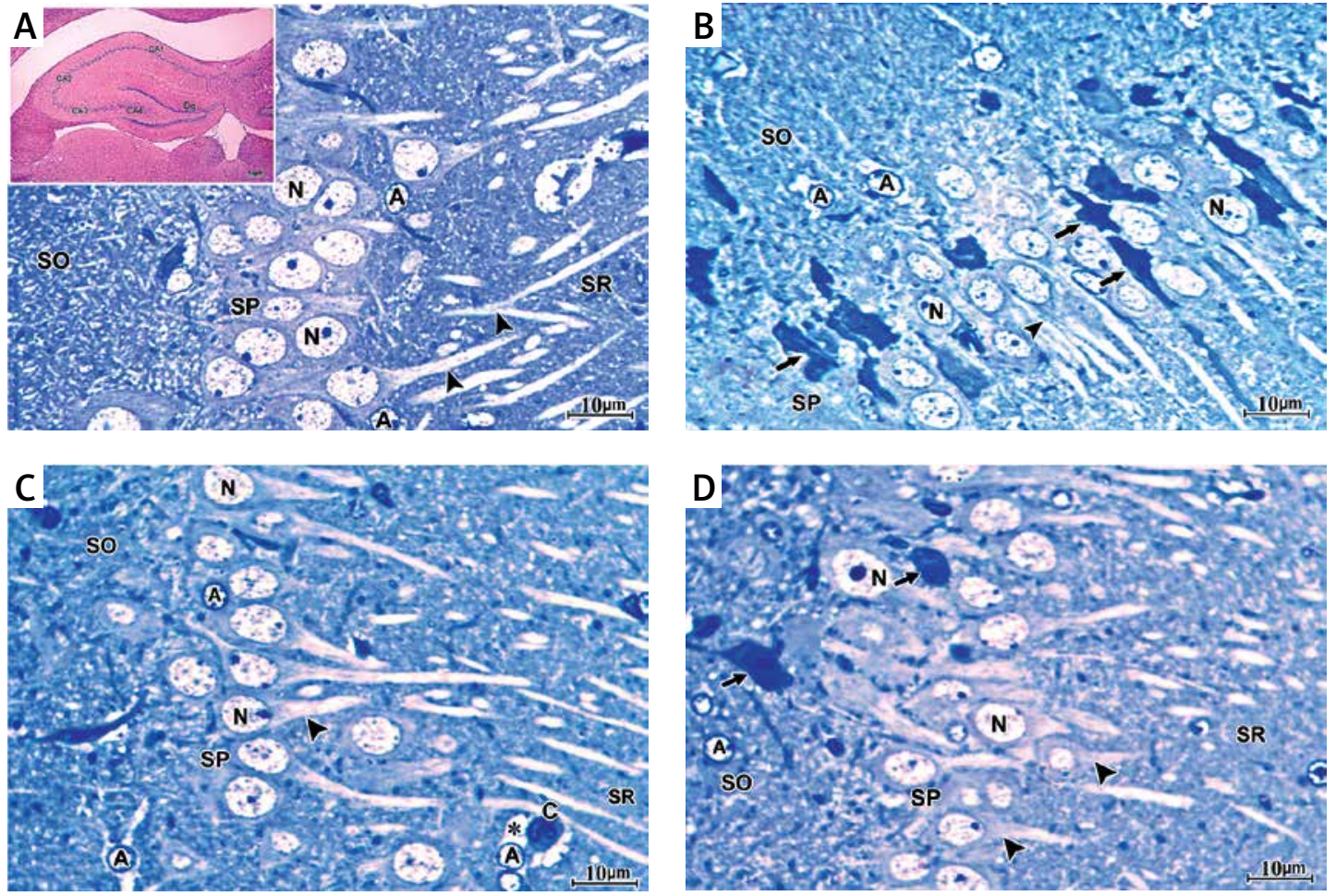

Figure 4. A - The semithin section of the CA1 field of control group showing large sized pyramidal neurons with large vesicular nuclei $(\mathrm{N})$ and considerable amount of basophilic granular cytoplasm closely packed in stratum pyramidale (SP) with their long prominent dendrites (arrowhead) projecting into stratum radiatum. Deep in the pyramidal cell layer is the stratum oriens. Scattered astrocytes (A) are observed in the CA1 field of the control hippocampus. They exhibit pale oval or rounded nuclei surrounded by a thin rim of lightly stained cytoplasm. The inset shows a photomicrograph of a section of hippocampus of the control group stained with H\&E stain showing the dentate gyrus (Dg), Ammons horn or cornu ammonis (CA) with its subdivisions CA1, CA2, CA3 and CA4 fields. B - Semithin section of CA1 field of the hippocampus of APAP-E group showing numerous irregular darkly stained pyramidal neurons with pyknotic nuclei (arrow) and long dark dendrites (arrowhead). Other neurons have large vesicular nuclei $(\mathrm{N})$. Note the presence of numerous astrocytes $(\mathrm{A})$ with thin processes extending from their cell bodies. C - Semithin section of CA1 field of the hippocampus of coenzyme Q10-treated group showing most of the pyramidal neurons in SP appears normal with large vesicular nuclei $(\mathrm{N})$ and prominent nucleolus with long dendrite (arrowhead). Note that the astrocytes (A) have dilated processes $\left(^{*}\right)$ and surround the blood capillary (C). D - Semithin section of CA1 field of the hippocampus of captopril-treated group showing most of the pyramidal neurons in SP appears normal with large vesicular nuclei $(\mathrm{N})$ and prominent nucleoli with long dendrite (arrowhead). Few cells are shrunken with darkly stained cytoplasm and pyknotic nuclei (arrow). Scale bar $10 \mu \mathrm{m}$ and direct magnification 1000

large vesicular nuclei, prominent nucleoli and long dendrites. A few cells were shrunken with darkly stained cytoplasm and pyknotic nuclei (Figure 4 D).

\section{Immunohistochemistry}

Immunostaining with GFAP for detection of astrocytes in the control group showed scattered distribution of astrocytes which appeared brown in all the layers of the CA1 field. The astrocytes appeared stellate in shape with positive scanty cytoplasm and thin long branching processes. They appeared surrounding the blood capillaries (Figure $5 \mathrm{~A}$ ). In the APAP-E group, immunostaining for GFAP revealed the presence of numerous strongly positive astrocytes and thick profusely branching processes distributed in all layers of CAI (Figure 5 B). As regards CoQ10- and captopril-treated groups, there were numerous immunopositive astrocytes with long thin processes and surrounding blood capillary (Figures 5 C, D); however, they were less numerous compared to the APAP-E group.

\section{Electron microscopy}

Electron micrograph of the control CA1 field showed pyramidal neurons which were uniform in size with large rounded euchromatic nuclei with finely dispersed chromatin and prominent nucleoli. The surrounding cytoplasm contained numerous rough endoplasmic reticulum, free ribosomes and few lysosomes (Figures 6 A, B, D). A large dendrite containing microtubules and mitochondria was present (Figure $6 \mathrm{C}$ ). The astrocyte had a large oval euchromatic nucleus and intact mitochondria (Figure 6 D). A blood capillary with a wide lumen lined with endothelial cells and surrounded by pericytes was also observed (Figure 6 C). 

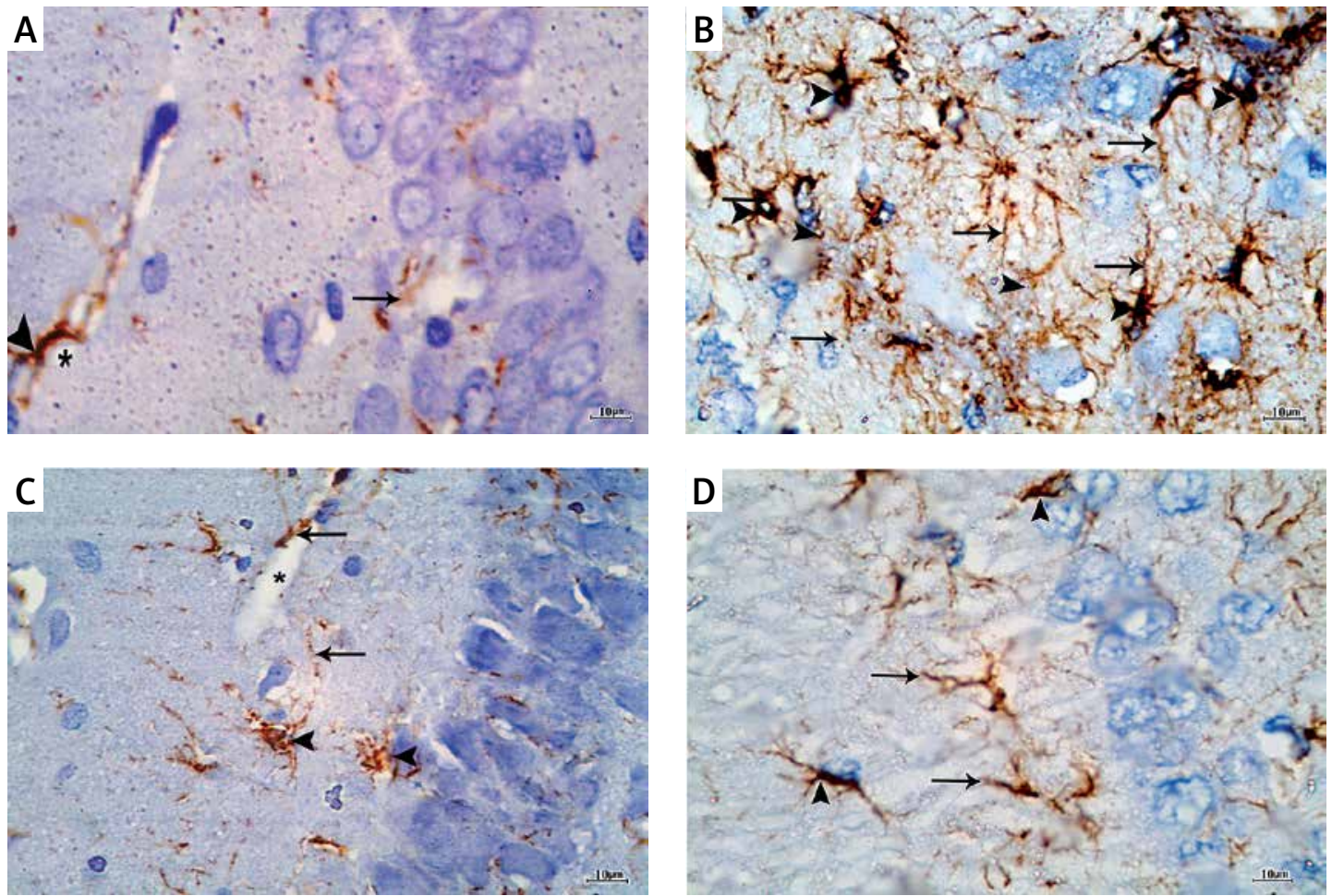

Figure 5. A - Immunostained section with glial fibrillary acid protein in the control group showing scattered positively stained astrocytes in all layers of CA1 field (arrowhead) with thin long processes (arrow) surrounding blood capillaries $\left({ }^{\star}\right)$. B - Autophagy protects against acetaminophen induced encephalopathy group immunostained section showing increased number of highly positively stained astrocytes (arrowhead) in all layers with prominent long branching processes (arrow). C - In coenzyme O10-treated group, there are strongly immunopositive astrocytes (arrowhead) and long thin processes (arrow) and surround blood capillary $\left({ }^{*}\right)$. D - the captopril-treated group showing numerous immunopositive astrocytes (arrowhead) and long thin processes (arrow). Scale bar $10 \mu \mathrm{m}$ and direct magnification 1000

In the APAP-E group, the CA1 field showed irregular electron-dense degenerating pyramidal neurons with ill-defined nuclei scattered among normal electron-lucent neurons, which had euchromatic nuclei and prominent nucleoli (Figure 7 A). These dark neurons were surrounded by dilated astrocytic processes (Figure 7 A). The cytoplasm exhibited swollen mitochondria with an electron-lucent matrix containing few cristae (Figure 7 B). Numerous large lysosome-like dense-bodies were present (Figures 7 B, C). Some dendrites were dark, while others were lightstained and revealed mitochondria as multiple individual teardrop-shaped organelles connected by thin membrane or mitochondria-on-a-string (MOAS) (Figures $7 \mathrm{~A}, \mathrm{D}$ ).

The astrocyte appeared with a heterochromatic nucleus having a prominent thick electron lucent processes surrounding the dark neurons and small blood capillary (Figures 7 A, E).

As regards the CoQ10-treated group, the pyramidal neurons appeared with large euchromatic nuclei and prominent nucleoli. The cytoplasm appeared translucent with several intact mitochondria and a huge amount of rER and large lysosome (Figures 8 A-C). The dendrite appeared normal, with well-organized microtubules and mitochondria (Figures 8 C, D). The astrocyte appeared with a rounded large euchromatic nucleus and prominent nucleolus. Its cytoplasm had short strands of rER and intact mitochondria (Figure $8 \mathrm{E}$ ).

In the captopril-treated group, multiple neurons appeared normal with rounded euchromatic nuclei and nucleoli. The cytoplasm had numerous slightly dilated $r E R$ and multiple lysosome-like dense bodies (Figures 9 A, B). The dendrite exhibited well-organized microtubules, some mitochondria appeared normal oval, while others appeared abnormally elongated (Figures 9 C, D). The astrocytes appeared with dark nuclei and wide astrocytic processes, which also surrounded the blood capillary (Figures 9 C, D).

\section{Morphometric and statistical results \\ Pyramidal cell thickness}

The thickness of the pyramidal cell layer is significantly decreased in APAP-E in comparison to control, CoQ10- and captopril-treated groups ( $p=0.002,0.002,0.009$; respectively). In contrast, administration of $\mathrm{CoQ} 10$ or captopril restored the normal thickness of the pyramidal cell layer with 

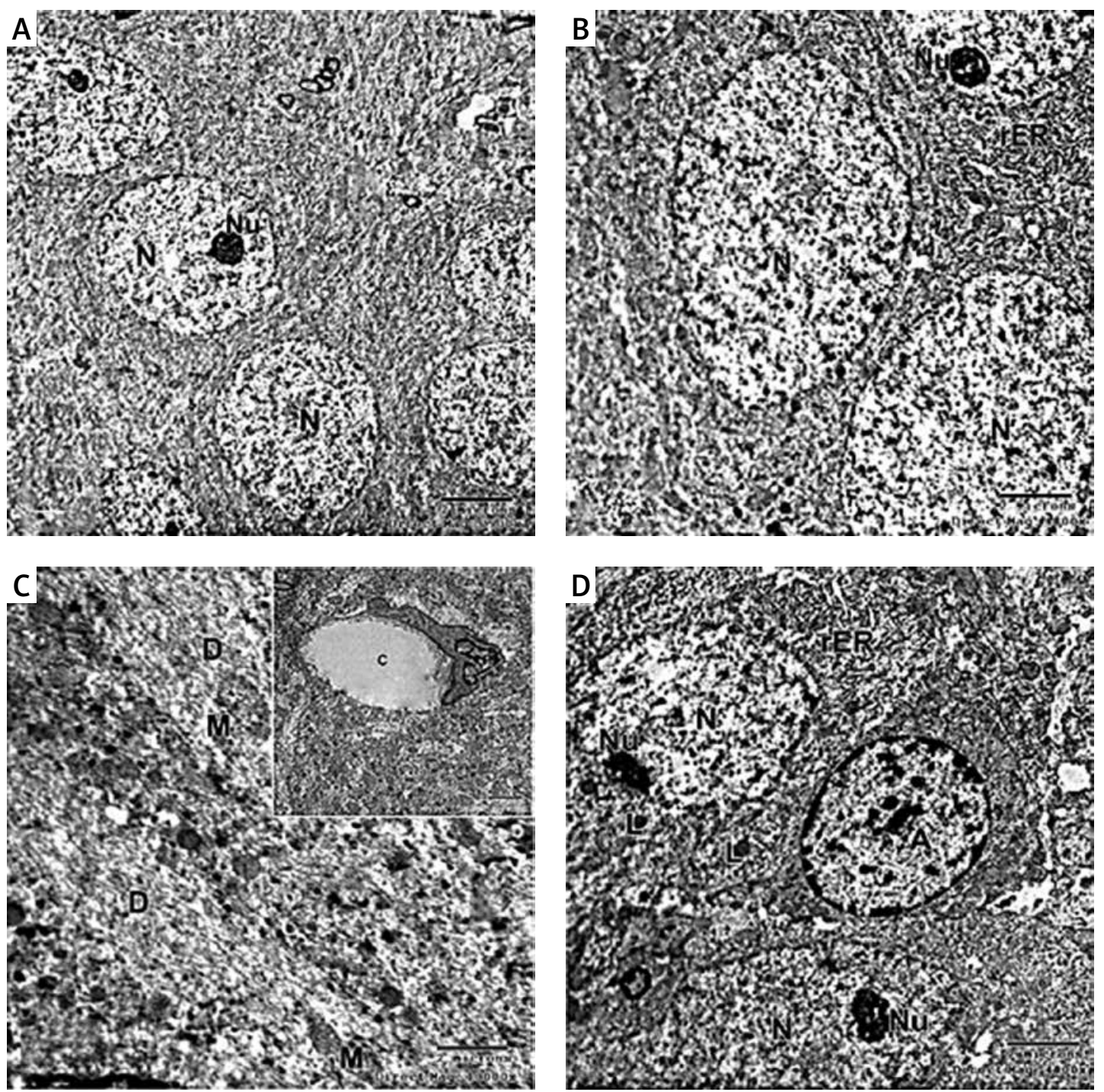

Figure 6. A - Electron micrograph of the control group CA1 field showing numerous pyramidal neurons with euchromatic nuclei $(\mathrm{N})$ and prominent nucleoli $(\mathrm{Nu})$. Scale bar $2 \mu \mathrm{m}$ and direct magnification 2900. B - A magnified part of control CA1 field showing the pyramidal neurons with euchromatic nuclei $(\mathrm{N})$, prominent nucleolus ( $\mathrm{Nu}$ ), and numerous rough endoplasmic reticulum (rER). Scale bar $2 \mu \mathrm{m}$ and direct magnification 4800. C- Electron micrograph of the control CA1 field showing dendrite (D) with well-organized microtubules and oval mitochondria (M). Scale bar $2 \mu \mathrm{m}$ direct and magnification 10000. The inset shows blood capillary with wide lumen surrounded by pericyte (P). Scale bar $2 \mu \mathrm{m}$ direct and magnification 5800. D - Electron micrograph of the control group CA1 field showing astrocyte (A) with large rounded nucleus, the cytoplasm surrounds the nucleus with short strands of $r E R$ and mitochondria $(M)$. Neurons with extensive rER and small lysosome-like dense bodies $(L)$ are present. Scale bar $2 \mu \mathrm{m}$ and direct magnification 4800

no significant difference from the control group (Figure $10 \mathrm{~A}$ ).

\section{Number of dark neurons}

The number of dark neurons increased significantly in APAP-E rats in comparison with the control group $(p=0.002)$. Meanwhile, the administration of CoQ10 or captopril reduced the number of dark neurons to a marked extent vs. APAP-E ( $p=0.002$ in both); however, the number was still significantly higher in the captopril-treated group vs. the control group ( $p=0.026)$ (Figure $10 \mathrm{~B}$ ).
Number of glial fibrillary acid protein astrocytes

Figure $10 \mathrm{C}$ shows that, in the APAP-E group, the number of GFAP-positive cells was significantly higher in comparison with the control, CoQ10- and captopril-treated groups ( $p=0.002,0.041,0.026$; respectively). Although oral intake of captopril reduced the number of GFAP-positive cells, it was still significantly higher than the control group $(p=0.002)$. To a much better extent, the administration of CoQ10 normalized the level of GFAP-positive cells with no significant difference from the control group. 

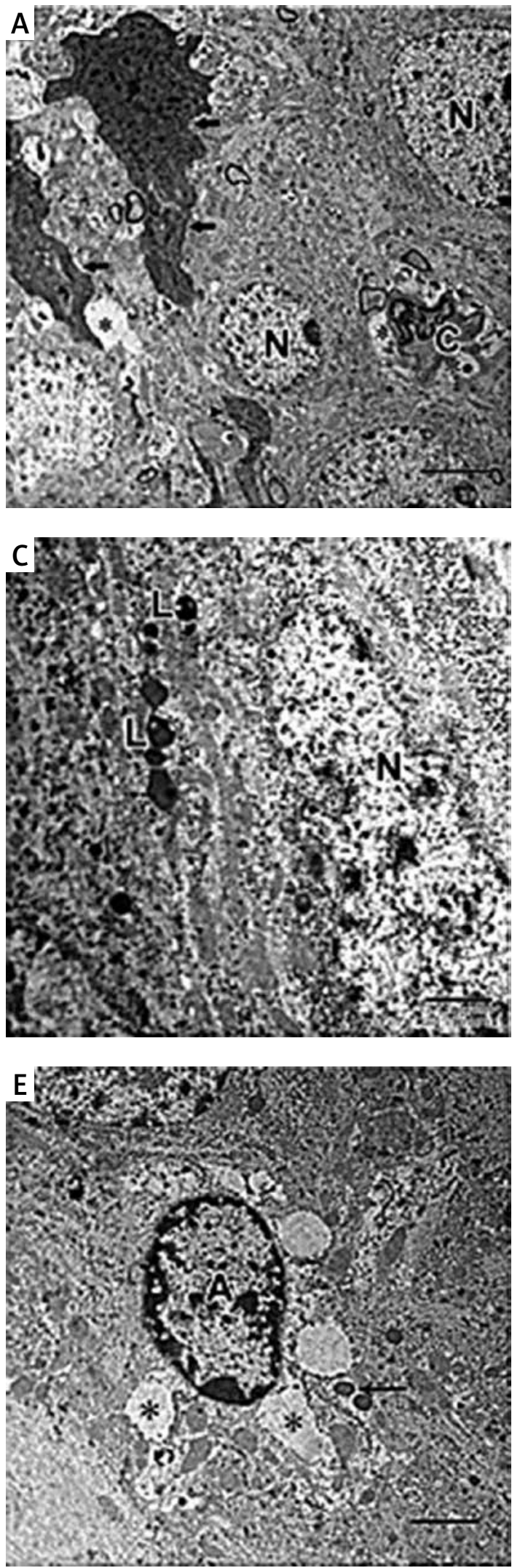
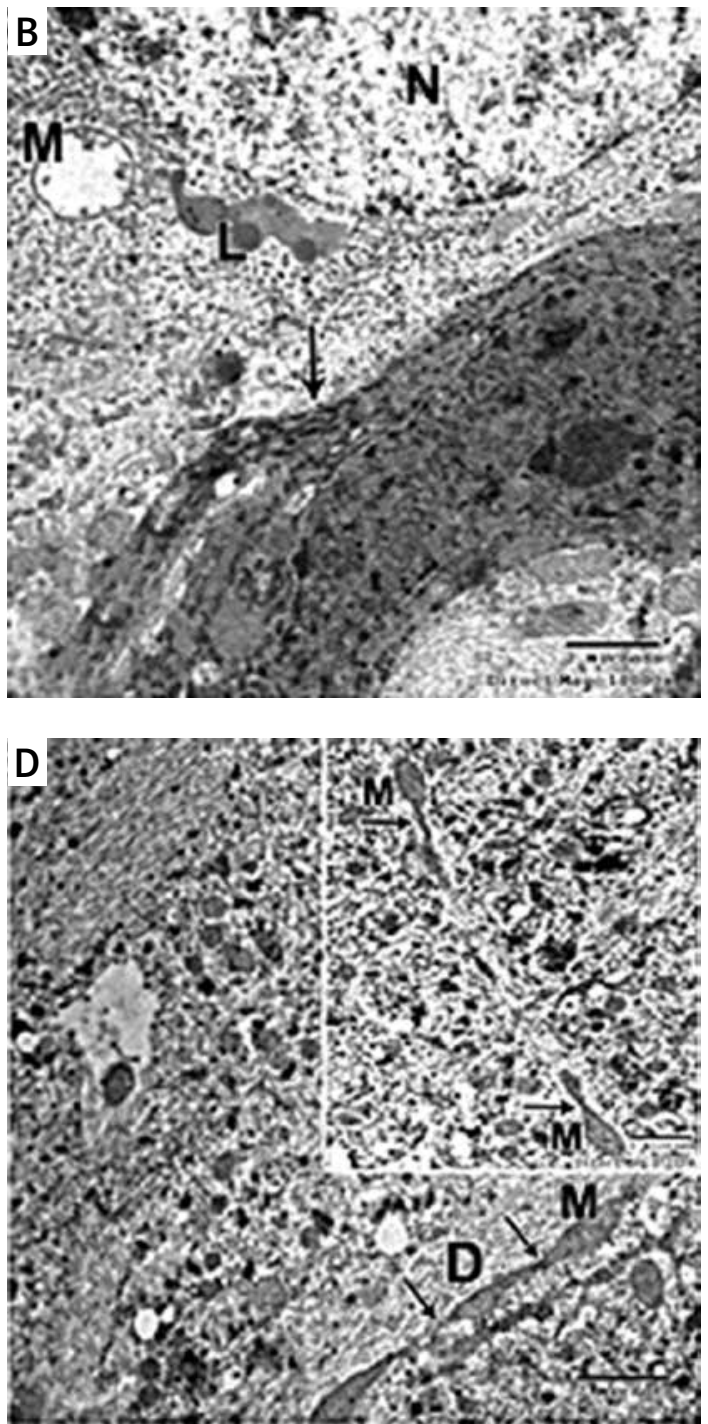

Figure 7. A - Electron micrograph of autophagy protects against acetaminophen induced encephalopathy group (APAP-E ) CA1 field showing electron dense irregular pyramidal neurons (thick arrow) with ill-defined nuclei and dark dendrite (arrowhead) among normal electron lucent neurons $(\mathrm{N})$. Note the dilated astrocytic processes $\left.{ }^{*}\right)$ surrounding the dark neurons and small blood capillary (C). Scale bar $2 \mu \mathrm{m}$ and direct magnification 2900. B - Electron micrograph of CA1field of APAP-E group showing part of neuron $(\mathrm{N})$ containing swollen mitochondria $(\mathrm{M})$ with electron lucent matrix containing a few cristae. Note the presence of numerous lysosomes (L). The arrow points to part of dark neuron with ill-defined nucleus. Scale bar $2 \mu \mathrm{m}$ and direct magnification 10000. C - Electron micrograph of APAP-E group CA1 field showing part of neuron with large irregular euchromatic nucleus (N) and numerous lysosomes (L). Scale bar $2 \mu \mathrm{m}$ and direct magnification 10000. D - Electron micrograph of APAP-E group CA1 field showing a dendrite (D) with elongated pointed end mitochondria $(M)$ interconnected by thin membranes (arrow) the inset showing interconnected mitochondria and mitochondria with long process. Scale bar $2 \mu \mathrm{m}$ and direct magnification 7200. E - Electron micrograph of CA1field of APAP-E showing astrocyte prominent thick electron lucent processes $\left({ }^{*}\right)$. Note the presence of numerous dense bodies (arrow). Scale bar $2 \mu \mathrm{m}$ and direct magnification 5800 

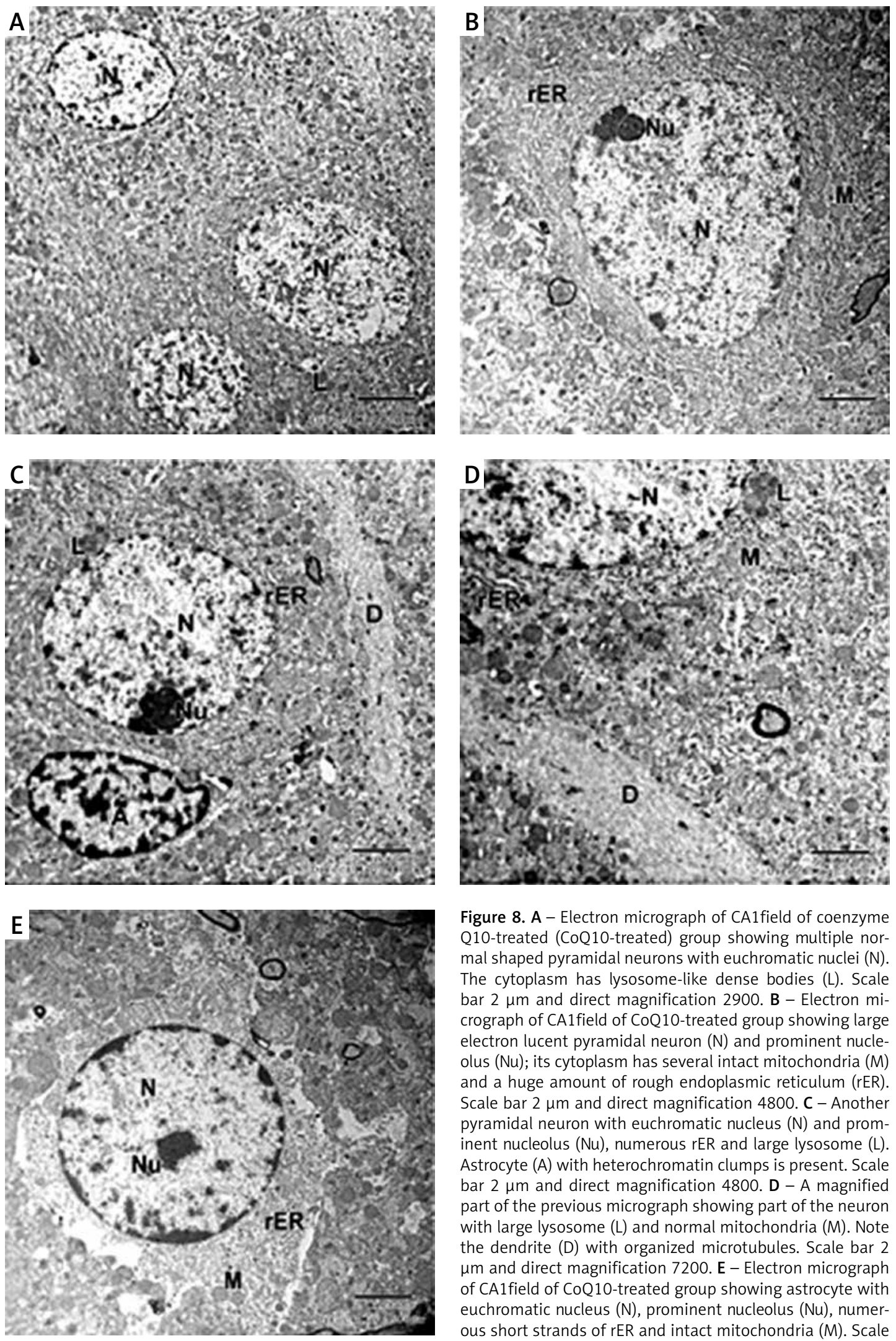

Figure 8. A - Electron micrograph of CA1field of coenzyme Q10-treated (CoQ10-treated) group showing multiple normal shaped pyramidal neurons with euchromatic nuclei $(\mathrm{N})$. The cytoplasm has lysosome-like dense bodies (L). Scale bar $2 \mu \mathrm{m}$ and direct magnification 2900. B - Electron micrograph of CA1field of CoQ10-treated group showing large electron lucent pyramidal neuron $(\mathrm{N})$ and prominent nucleolus ( $\mathrm{Nu}$ ); its cytoplasm has several intact mitochondria (M) and a huge amount of rough endoplasmic reticulum ( $\mathrm{rER}$ ). Scale bar $2 \mu \mathrm{m}$ and direct magnification 4800. C - Another pyramidal neuron with euchromatic nucleus $(\mathrm{N})$ and prominent nucleolus ( $\mathrm{Nu}$ ), numerous rER and large lysosome ( $\mathrm{L}$ ). Astrocyte (A) with heterochromatin clumps is present. Scale bar $2 \mu \mathrm{m}$ and direct magnification 4800. D - A magnified part of the previous micrograph showing part of the neuron with large lysosome (L) and normal mitochondria (M). Note the dendrite (D) with organized microtubules. Scale bar 2 $\mu \mathrm{m}$ and direct magnification 7200. E - Electron micrograph of CA1field of CoQ10-treated group showing astrocyte with euchromatic nucleus $(\mathrm{N})$, prominent nucleolus $(\mathrm{Nu})$, numerous short strands of $r E R$ and intact mitochondria (M). Scale bar $2 \mu \mathrm{m}$ and direct magnification 5800 

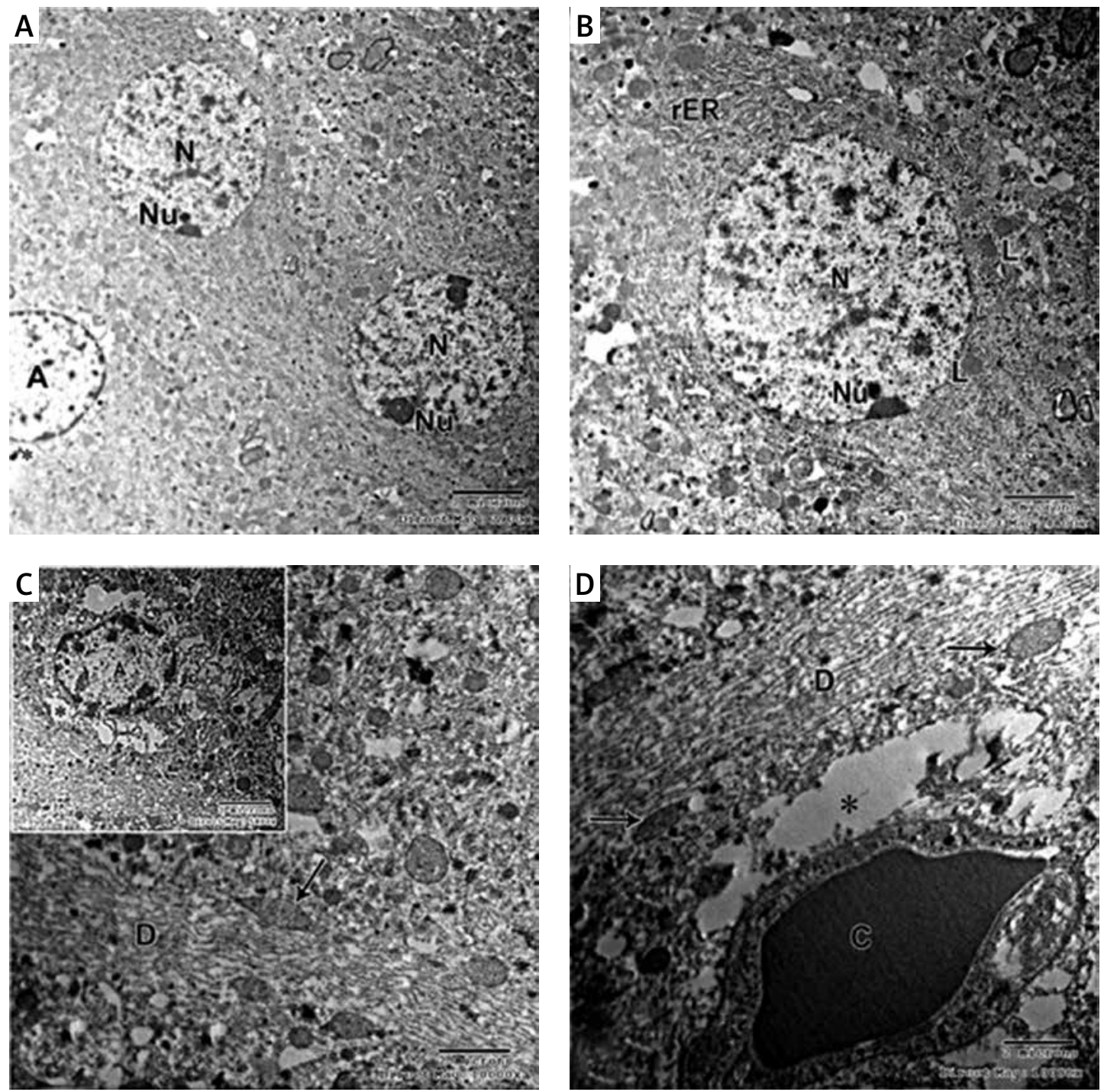

Figure 9. A - Electron micrograph of CA1field of captopril-treated group showing 2 pyramidal neurons that appear fully normal $(\mathrm{N})$ with rounded euchromatic nuclei and prominent nucleoli $(\mathrm{Nu})$. Part of astrocyte (A) with dilated electron-lucent process $\left(^{*}\right)$ is present. Scale bar $2 \mu \mathrm{m}$ and direct magnification 2900. B - A magnified part of the previous micrograph showing neuron with rounded euchromatic nucleus $(\mathrm{N})$ and nucleolus $(\mathrm{Nu})$. The cytoplasm has numerous slightly dilated (rough endoplasmic reticulum) and lysosomes (L). Scale bar $2 \mu \mathrm{m}$ and direct magnification 4800. C - Electron micrograph of part of a dendrite (D) showing abnormally shaped elongated pointed mitochondria (arrow). Scale bar $2 \mu \mathrm{m}$ and direct magnification 10000. The inset shows astrocyte (A) with wide electron lucent processes $\left({ }^{*}\right)$ and intact mitochondria (M). Scale bar $2 \mu \mathrm{m}$ and direct magnification 5800. D - Electron micrograph of CA1field of captopril-treated group showing blood capillary (C) surrounded by wide astrocytic processes ${ }^{\star}$ ). The dendrite has well-organized microtubules and normal shaped oval mitochondria (arrow). Scale bar $2 \mu \mathrm{m}$ and direct magnification 10000

\section{Discussion}

This study was designed to investigate the possible effects of CoQ10 and captopril against APAP-induced encephalopathy. The current study showed that rats with encephalopathy exhibited impaired behavioral testing evidenced by impaired intermediate and long-term memories in the NOR test, reduced locomotor activity in the activity cage, high serum ammonia levels, low serum TAC levels, and impaired $\mathrm{Na}^{+} / \mathrm{K}^{+}$ATPase activity, confirming the brain insult. It is well known that a high level of ammonia is toxic to the ner- vous system; it results in encephalopathy, and eventually coma and death [39]. Moreover, the hyperbilirubinemia that may be encountered in patients with hepatic encephalopathy induced impairment of memory through impairing synaptic plasticity in the hippocampus via disturbing longterm potentiation that facilitates information storage at the synaptic level and is considered the chief component of memory consolidation [40]. Acetaminophen can easily penetrate the blood brain barrier, activating the neuronal cytochrome P450 2E1, generating toxic metabolites such as 
A

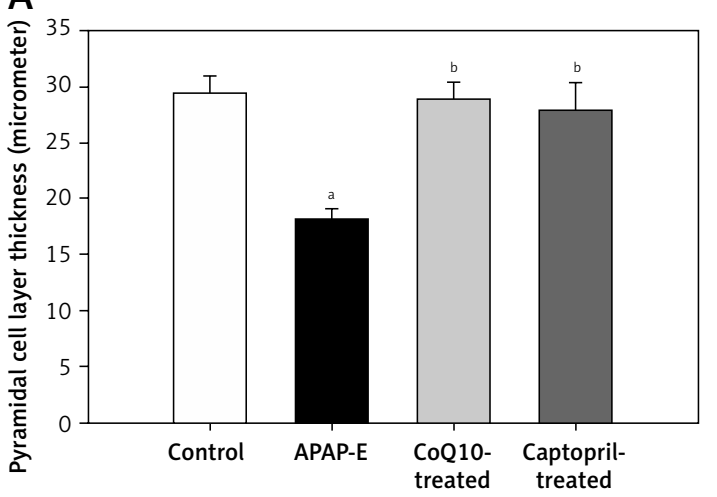

C

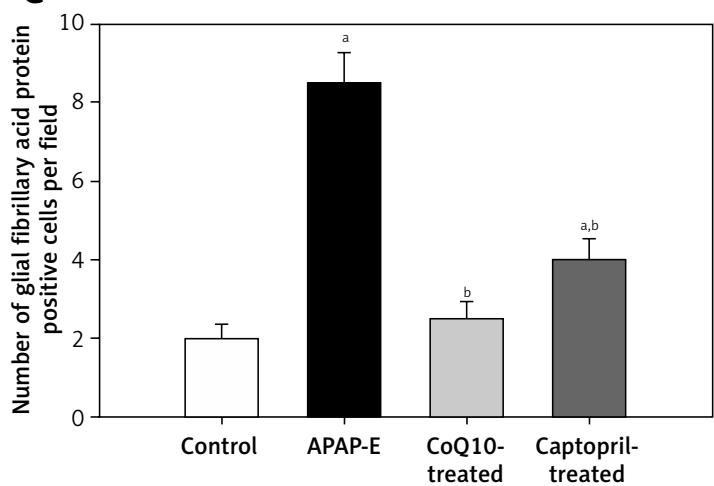

$\mathrm{N}$-acetyl-p-benzoquinoneimine (NAPQI), which decreases glutathione, ascorbic acid, superoxide dismutase levels, resulting in oxidative stress and neurotoxicity [3]. Oxidative stress results in cellular damage, mitochondrial dysfunction and inhibition of $\mathrm{Na}^{+} / \mathrm{K}^{+}$ATPase [41].

$\mathrm{Na}^{+} / \mathrm{K}^{+}$ATPase, an essential integral protein in brain cells, is accountable for approximately half of total brain energy consumption [42]. It regulates the activity of gamma amino butyric acid [43] and glutamate transporters [44]. $\mathrm{Na}^{+} / \mathrm{K}^{+}$ ATPase dysfunction resulted in the inflow of $\mathrm{Na}^{+}$, $\mathrm{Ca}^{2+}, \mathrm{Cl}^{-}$ions and water and outflow of $\mathrm{K}^{+}$ions, which leads to cerebral edema [45]. Our results showed a reduction in brain $\mathrm{Na}^{+} / \mathrm{K}^{+}$ATPase activity in APAP-E group. Trumper et al. [46] reported that APAP overdose in rats (single $1000 \mathrm{mg} / \mathrm{kg}$ IP) results in a reduction in $\mathrm{Na}^{+} / \mathrm{K}^{+}$ATPase activity in renal proximal tubules. This reduction could be attributed to high ammonia levels, which activate the $\mathrm{N}$-methyl-D-aspartate glutamate receptor, resulting in increased protein kinase C-mediated inactivation of $\mathrm{Na}^{+} / \mathrm{K}^{+}$ATPase via phosphorylation [23].

Microtubule-associated protein light chain-3 (LC3-II) and beclin-1 are the most trustworthy cellular markers for autophagosome formation and autophagy [6]. Chao et al. [47] reported that APAP overdose activates liver autophagy and agents that induce autophagy may be protective against APAP-induced hepatotoxicity. Moreover, Soria et

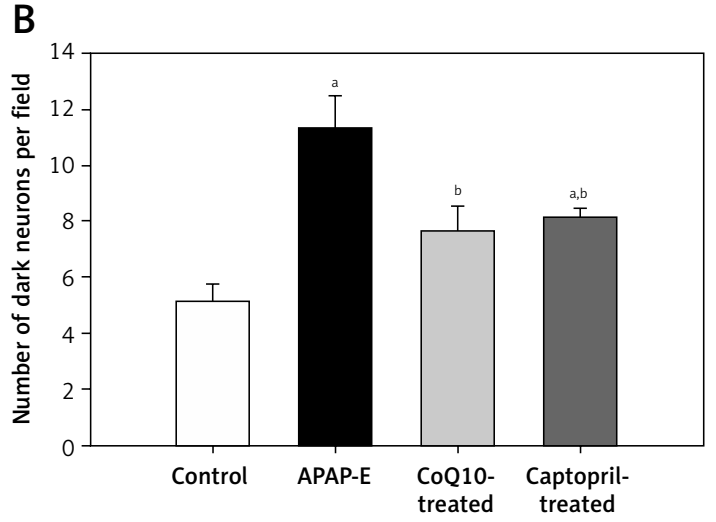

Figure 10. Pyramidal cell thickness in $\mu \mathrm{m}$, number of dark neurons, number of glial fibrillary acidic protein positive astrocytes in CA1 field of hippocampus. Data are shown as means \pm standard error of the mean ( $n=6$ in each group). aSignificant as compared to the control group $p<0.05$. bSignificant as compared to the autophagy protects against acetaminophen induced encephalopathy group $P<0.05$. The Kruskal-Wallis $\mathrm{H}$ test followed by the Mann-Whitney U test was used for statistical analysis

al. [48] stated that high levels of ammonia induce hepatic autophagy, resulting in urea synthesis and ammonia detoxification. In line with this, our results showed an increase in the expression levels of hippocampal LC3-II and beclin-1 transcripts in rats with APAP-induced encephalopathy - an attempt to minimize the brain insult. Additionally, high levels of ammonia reduced the antioxidant capacity and resulted in mitochondrial dysfunction and inactivation of hippocampal $\mathrm{Na}^{+} / \mathrm{K}^{+} \mathrm{AT}-$ Pase.

Rats treated with either $\mathrm{CoQ} 10$ or captopril, in the present study, showed an improvement in behavioral tests proved by potentiation of the intermediate and long-term memories in the NOR test measured after 4 and 24 hours respectively, increased locomotor activity associated with lower levels of ammonia, higher TAC levels and higher $\mathrm{Na}^{+} / \mathrm{K}^{+}$ATPase activity. Fouad and Jresat [49] reported that CoQ10 attenuated APAP-induced hepatotoxicity via inhibiting lipid peroxidation and inducible nitric oxide (NO) synthase, thus decreasing NO production and preventing NO-induced depletion of glutathione and catalase. Al-Shaikh et al. [50] reported that captopril given to rats (20 $\mathrm{mg} / \mathrm{kg} /$ day orally for 10 days) has hepatoprotective effects against APAP overdose, which might be attributed to its sulfhydryl group that is not present in other ACE inhibitors that acts as a free radical scavenger. In addition, captopril is reported to ameliorate oxidative stress by enhancing total 
glutathione content as well as glutathione peroxidase and glutathione reductase activities in various mouse tissues [51]. In the current experiment, decreased brain Ang II activity after captopril administration improved cognitive performance probably by enhancing CGMP formation and the subsequent release of glutamate that potentiates synaptic plasticity, a well-known neuronal mechanism in the learning and memory processes [52]. Furthermore, CoQ10 and captopril enhance autophagy as evidenced by increased expression levels of LC3-II and beclin-1 transcripts and thus could be beneficial against APAP-induced encephalopathy. Mohamed et al. [53] found that administration of CoQ10 injected IP in a dose of $4 \mathrm{mg} / \mathrm{kg} /$ day for 4 weeks attenuated methotrexate induced-lung and liver tissue injury via up-regulating the expression of autophagy-related genes such as LC3B. Further activation of autophagy by either CoQ10 or captopril administration reduced ammonia levels and thus restored antioxidant capacity and hippocampal $\mathrm{Na}^{+} / \mathrm{K}^{+}$ATPase activity.

In this study, we focused on the pyramidal neurons of AH's CA1 region, which is supposed to be the last element in the trisynaptic circuit for the passage of information in the hippocampus [54]. In APAP-E rats, these neurons showed variable degrees of structural alterations that appeared in the form of a significant reduction in the thickness of pyramidal cell layer together with the elevation of the number of abnormal shrunken dark irregular neurons containing poorly defined nuclei and electron-dense cytoplasm. A prominent manifestation of nerve injury following several brain insults is the occurrence of dark neurons which may progress to death [55]. These results are compatible with the findings of Posadas et al. [2], who described an injurious impact of acetaminophen on brain neurons acting via increased accumulation of the toxic metabolite NAPQI that consumes hippocampal glutathione content, leading to neuronal death in vivo as well as in vitro.

Structural alterations of the mitochondria were a striking feature in many neurons and neuronal processes in the APAP-E group. Some neurons showed swollen mitochondria with an electron-lucent matrix containing few cristae. Reactive oxygen species attack neurons, which are post-mitotic cells that are sensitive to the deleterious effect of free radicles leading to neuronal damage [56] and apoptosis in human neurons [57]. Structural changes of the mitochondria could be due to augmented oxidative stress, as evidenced by reduced serum TAC observed in this study. This may be explained by oxidative damage induced by acetaminophen that can affect several structural and functional components of the mitochondria, including mitochondrial DNA (mtDNA), proteins and membranes, leading to mitochondrial swelling and uncoupling of oxidative phosphorylation, which eventually results in ATP exhaustion and cell death [58]. It is well known that the disruption of mitochondrial function in neurons is typically associated with the initiation or augmentation of neuronal injury, so neuron degeneration in this study can be explained by oxidative damage and the accompanying mitochondrial dysfunction.

These abnormal damaged swollen mitochondria may be targeted for disposal by mitophagy, a mechanism of cellular homeostasis that eradicates injured mitochondria from cells in a quick and targeted manner [59] as evidenced by numerous lysosomes noted in the present study. The remnants of dysfunctional mitochondria may contribute to excessive generation of ROS [60], which might induce mitophagy that in turn eliminates the damaged organelles [61].

Another interesting finding was the existence of numerous abnormally shaped mitochondria that appeared as elongated multiple individual teardrop shaped organelles interconnected by thin membranes in the neuropils. This observation was reported by Trushina [62], who observed it in many conditions including Alzheimer's disease, hypoxia and aging and called it MOAS, which are formed during the final steps of the fission process of the mitochondria. Fission process of the mitochondria is vital for the quality control maintaining healthy mitochondria [63]. This delayed fission during neurodegenerative diseases indicates the compensatory adaptation of mitochondria to oxidative stress [62].

Moreover, in the APAP-E group, the neurons contained numerous lysosomes of variable sizes. The appearance of lysosomes in abundant numbers in the hippocampal CA1 neurons at different stages of maturation may indicate activation of lysosomal protein degradation in a trial for protecting the cells from further structural damage.

In this study, there was a marked increase in astrocytes in the APAP-E group as evidenced by the marked rise in the number of GFAP-positive cells in all layers of the CA1 field; GFAP is considered to be the main constituent of the intermediate astrocyte filaments. The ultrastructure of astrocytes exhibited wide processes surrounding the degenerated neurons and blood capillaries. In response to various central nervous system (CNS) pathologies, astrocytes become active, resulting in a process called "reactive gliosis," a process with specific structural and functional features [64]. Increased GFAP expression is associated with hyper-reactivity and altered morphology of the astrocyte [65]. An important function of activated or reactive astrocytes is the preservation of the nervous tissue integrity following injury, acting as 
a defense mechanism to minimize and repair the initial damage after CNS injuries. When they enter a reactive state, they are morphologically characterized by increases of cell size and number and the proliferation and enlargement of cellular processes [66]. Astrocytes support neuronal function by producing antioxidants (glutathione), recycling neurotransmitters such as glutamate and gamma-aminobutyric acid, and sustain the microenvironmental equilibrium surrounding neurons and in whole tissue homeostasis [67]; astrocytes support these critical functions through specialized responses to stress or toxic conditions [68]. Astrocytes also act on the brain by clearing ROS and excess glutamate from the local environment [69]. So their reduction after administration of CoQ10 and captopril may be due to the decrease of ROS and glutamate.

As regards the histological results of the CoQ10-treated group, there was a substantial improvement as the majority of the pyramidal neurons appeared normal, containing large euchromatic nuclei, translucent cytoplasm, with restoration of normal appearance of the mitochondria. The dark neurons were significantly reduced in number, and the thickness of the SP layer in the CA1 field was significantly increased. This is in agreement with Won et al. [70], who reported that treatment with $\mathrm{CoQ} 10$ reduced neuronal cell death. This could be due to the ability of CoQ10 to stabilize mitochondrial calcium-dependent ion channels and reduce cell energy depletion [71]. Moreover, it is considered as a potent free radical scavenger in lipid and mitochondrial membranes [72]; thus, CoQ10 can show efficacy in treating patients with known mitochondrial disorders [73]. Our results showed that astrogliosis was reduced in the CoQ10-treated group, which could be explained by the results of Lee et al. [74], who suggested that CoQ10 may provide neuroprotection against ischemic retinal injury via reduction of oxidative stress, which in turn blocks activation of astroglial and microglial cells in the ischemic retina.

It was demonstrated that CoQ10 by its antioxidant, anti-inflammatory, and anti-apoptotic effects can play a therapeutic role in APAP hepatotoxicity, metabolic-stress-induced liver damage [16], and acute myocardial ischemia-reperfusion injury [75].

In the captopril treated group, there was restoration of the normal appearance of most neurons and a reduction in the number of dark cells and increase of the pyramidal cell thickness. Ultrastructurally, some mitochondria in the dendrites appeared elongated with pointed ends. Astrocytes were reduced in number compared to the APAP-E group but still high and appeared heterochromatic with wide astrocytic processes while others appeared euchro- matic. Captopril has been shown to protect against acute and chronic Parkinson disease besides its modification of astrocytic function and activation of glial cells in the substantia nigra of the rat model for Parkinson disease [76]. Captopril functions as an antioxidant both by scavenging ROS due to its thiol group [77] and by enhancing the activities of antioxidant enzymes such as superoxide dismutase and glutathione peroxidase [61].

In conclusion, oral administration of CoQ10 as well as captopril for 7 days reduced oxidative stress and enhanced neuronal autophagy and as a result reduced ammonia and its inhibitory impact on $\mathrm{Na}^{+} / \mathrm{K}^{+}$ATPase activity, thereby playing a pivotal role in protecting neurons against APAP-induced encephalopathy. This study offers new therapeutic targets for APAP-induced encephalopathy and enhances our understanding of the clinical applications of CoQ10 and captopril. The therapeutic role and the underlying mechanisms of possible combinations of CoQ10 and captopril against APAP-induced encephalopathy should be addressed in future studies.

\section{Conflict of interest}

The authors declare no conflict of interest.

\section{References}

1. Shabrang B, Jamshidzadeh A, Farjam M, Ebrahimpour A, Koohi-Hosseinabadi O. Concurrent assessment of calpain and caspase 3 activities in brains of mice with acetaminophen-induced acute hepatic encephalopathy. Metab Brain Dis 2017; 32: 2139-42.

2. Posadas I, Santos P, Blanco A, Munoz-Fernandez M, Cena V. Acetaminophen induces apoptosis in rat cortical neurons. PLoS One 2010; 5: e15360.

3. Essawy AE, Alkhuriji AF, Soffar AA. Paracetamol overdose induces physiological and pathological aberrations in rat brain. J Appl Pharm Sci 2017; 7: 185-90.

4. Pandurangan AK, Ismail S, Esa NM, Munusamy MA. Inositol-6 phosphate inhibits the mTOR pathway and induces autophagy-mediated death in HT-29 colon cancer cells. Arch Med Sci 2018; 14: 1281-8.

5. Kiffin R, Bandyopadhyay U, Cuervo AM. Oxidative stress and autophagy. Antioxid Redox Signal 2006; 8: 152-62.

6. Ni H, Gong Y, Yan JZ, Zhang LL. Autophagy inhibitor 3-methyladenine regulates the expression of LC3, Beclin-1 and ZnTs in rat cerebral cortex following recurrent neonatal seizures. World J Emerg Med 2010; 1: 216-23.

7. Mizushima N, Levine B, Cuervo AM, Klionsky DJ. Autophagy fights disease through cellular self-digestion. $\mathrm{Na}$ ture 2008; 451: 1069-75.

8. Ni HM, Bockus A, Boggess N, Jaeschke H, Ding WX. Activation of autophagy protects against acetaminopheninduced hepatotoxicity. Hepatology 2012; 55: 222-32.

9. Moazen M, Mazloom Z, Dabbaghmanesh MH, Ahmadi A. Effect of CoQ10 supplementation on blood pressure, inflammation, and lipid profile in type 2 diabetics. Iran J Nutr Sci Food Technol 2013; 8: 145-53.

10. Alam MA, Rahman MM. Mitochondrial dysfunction in obesity: potential benefit and mechanism of Co-enzyme 
Q10 supplementation in metabolic syndrome. J Diabetes Metab Disord 2014; 13: 60.

11. Qu H, Guo M, Chai H, et al. Effects of coenzyme Q10 on statin-induced myopathy: an updated meta-analysis of randomized controlled trials. J Am Heart Assoc 2018; 7: e009835.

12. Mazidi M, Kengne AP, Banach M. Effects of coenzyme Q10 supplementation on plasma C-reactive protein concentrations: a systematic review and meta-analysis of randomized controlled trials. Pharmacol Res 2018; 128: 130-6.

13. Stroes ES, Thompson PD, Corsini A, et al. Statin-associated muscle symptoms: impact on statin therapy - European Atherosclerosis Society consensus panel statement on assessment, aetiology and management. Eur Heart J 2015; 36: 1012-22.

14. Ashby EL, Kehoe PG. Current status of renin-aldosterone angiotensin system-targeting anti-hypertensive drugs as therapeutic options for Alzheimer's disease. Expert Opin Investig Drugs 2013; 22: 1229-42.

15. Zhang X, Liu H, Hao Y, et al. Coenzyme Q10 protects against hyperlipidemia-induced cardiac damage in apolipoprotein E-deficient mice. Lipids Health Dis 2018; 17 279

16. Vasiliev AV, Martinova EA, Sharanova NV, Gapparov MM Effects of coenzyme Q10 on rat liver cells under conditions of metabolic stress. Bull Exp Biol Med 2011; 150 416-9.

17. Bodiga VL, Bodiga S. Renin angiotensin system in cognitive function and dementia. Asian J Neurosci 2013 2013: 1-18.

18. O'Caoimh R, Kehoe PG, Molloy DW. Renin Angiotensin aldosterone system inhibition in controlling dementia-related cognitive decline. J Alzheimers Dis 2014; 42 S575-86.

19. Onaolapo OJ, Adekola MA, Azeez TO, Salami K, Onaolapo AY. I-Methionine and silymarin: A comparison of prophylactic protective capabilities in acetaminophen-induced injuries of the liver, kidney and cerebral cortex. Biomed Pharmacother 2017; 85: 323-33.

20. Abd Allah ES, Gomaa AM. Effects of curcumin and cap topril on the functions of kidney and nerve in streptozotocin-induced diabetic rats: role of angiotensin converting enzyme 1. Appl Physiol Nutr Metab 2015; 40: 1061-7.

21. Ashkani Esfahani S, Esmaeilzadeh E, Bagheri F, Emami Y, Farjam M. The effect of co-enzyme q10 on acute liver damage in rats, a biochemical and pathological study. Hepat Mon 2013; 13: e13685.

22. Guide for the Care and Use of Laboratory Animals. $8^{\text {th }}$ Ed. National Academy Press, Washington, D.C., 2011.

23. Akkerman S, Blokland A, Reneerkens $O$, et al. Object recognition testing: methodological considerations on exploration and discrimination measures. Behav Brain Res 2012; 232: 335-47.

24. Antunes M, Biala G. The novel object recognition mem ory: neurobiology, test procedure, and its modifications. Cogn Process 2012; 13: 93-110.

25. Woode E, Amidu N, Owiredu WKBA, et al. Anxiogenic-like effects of a root extract of sphenocentrum jollyanum pierre in murine behavioural models. J Pharma Toxicol 2009; 4: 91-106.

26. Koracevic D, Koracevic G, Djordjevic V, Andrejevic S, Cosic V. Method for the measurement of antioxidant activity in human fluids. J Clin Pathol 2001; 54: 356-61.

27. Sehirli O, Sener E, Cetinel S, et al. Alpha-lipoic acid protects against renal ischemia-reperfusion injury in rats. Clin Exp Pharmacol Physiol 2008; 35: 249-55.
28. Atkinson A, Gatenby AD, Lowe AG. The determination of inorganic orthophosphate in biological systems. Biochim Biophys Acta 1973; 320: 195-204.

29. Lowry OH, Rosebrough NJ, Farr AL, Randall RJ. Protein measurement with the folin phenol reagent. J Biol Chem 1951; 193: 265-75.

30. Zhu B, Zheng YF, Zhang YY, et al. Protective effect of $\mathrm{L}$-carnitine in cyclophosphamide-induced germ cell apoptosis. J Zhejiang Univ Sci B 2015; 16: 780-7.

31. Miyatake $Y$, Ikeda $H$, Ishizu A, et al. Role of neuronal interferon-gamma in the development of myelopathy in rats infected with human T-cell leukemia virus type 1. Am J Pathol 2006; 169: 189-99.

32. Navarro VM, Castellano JM, Fernandez-Fernandez R, et al. Effects of KiSS-1 peptide, the natural ligand of GPR54, on follicle-stimulating hormone secretion in the rat. Endocrinology 2005; 146: 1689-97.

33. Jin BK, Shin DY, Jeong MY, et al. Melatonin protects nigral dopaminergic neurons from 1-methyl-4-phenylpyridinium (MPP+) neurotoxicity in rats. Neurosci Lett 1998; 245: 61-4.

34. Bancroft JD, Gamble M. Theory and Practice of Histological Techniques. Churchill Livingstone, Scotland, London, 2008.

35. Reilly JF, Maher PA, Kumari VG. Regulation of astrocyte GFAP expression by TGF-beta1 and FGF-2. Glia 1998; 22: 202-10.

36. Cattoretti G, Pileri S, Parravicini C, et al. Antigen unmasking on formalin-fixed, paraffin-embedded tissue sections. J Pathol 1993; 171: 83-98.

37. Gupta PD. Ultrastructural study of semithin section. Sci Tools 1983; 30: 6-7.

38. Knapp GR, Miller MC. Tests of Statistical Significance: Regression and Correlation. Williams \& Wilkins 1992, Baltimore, Maryland

39. Chen XL, Wee NL, Hiong KC, et al. Properties and expression of $\mathrm{Na}+\mathrm{K}+$-ATPase alpha-subunit isoforms in the brain of the swamp eel, Monopterus albus, which has unusually high brain ammonia tolerance. PLoS One 2013; 8: e84298.

40. Yang L, Wu D, Wang B, et al. The effects of hyperbilirubinaemia on synaptic plasticity in the dentate gyrus region of the rat hippocampus in vivo. Arch Med Sci 2020; 16: 200-4.

41. Sheu JJ, Sung PH, Leu S, et al. Innate immune response after acute myocardial infarction and pharmacomodulatory action of tacrolimus in reducing infarct size and preserving myocardial integrity. J Biomed Sci 2013; 20: 82.

42. Erecinska M, Silver IA. Ions and energy in mammalian brain. Prog Neurobiol 1994; 43: 37-71.

43. de Lores Arnaiz GR, Ordieres MG. Brain Na(+), K(+)-ATPase activity in aging and disease. Int J Biomed Sci 2014; 10: 85-102.

44. Sheean RK, Lau CL, Shin YS, O'Shea RD, Beart PM. Links between L-glutamate transporters, $\mathrm{Na+} / \mathrm{K}+-\mathrm{ATP}$ ase and cytoskeleton in astrocytes: evidence following inhibition with rottlerin. Neuroscience 2013; 254: 335-46.

45. Khanna A, Kahle KT, Walcott BP, Gerzanich V, Simard JM. Disruption of ion homeostasis in the neurogliovascular unit underlies the pathogenesis of ischemic cerebral edema. Transl Stroke Res 2014; 5: 3-16.

46. Trumper L, Coux G, Elias MM. Effect of acetaminophen on $\mathrm{Na}(+), \mathrm{K}(+)$ ATPase and alkaline phosphatase on plasma membranes of renal proximal tubules. Toxicol Appl Pharmacol 2000; 164: 143-8.

47. Chao X, Wang H, Jaeschke H, Ding WX. Role and mechanisms of autophagy in acetaminophen-induced liver injury. Liver Int 2018; 38: 1363-74. 
48. Soria LR, Allegri G, Melck D, et al. Enhancement of hepatic autophagy increases ureagenesis and protects against hyperammonemia. Proc Natl Acad Sci U S A 2018; 115: 391-6.

49. Fouad AA, Jresat I. Hepatoprotective effect of coenzyme Q10 in rats with acetaminophen toxicity. Environ Toxicol Pharmacol 2012; 33: 158-67.

50. Al-Shaikh TM, Mudawi MME, Yassin AYA, Habeballa RS, Chidrawar VR. Hepatoprotective Effect of captopril on liver toxicity induced by high and low dose of paracetamol in rats: histological study. Asian J Pharm Res Health Care 2016; 8: 92-9.

51. de Cavanagh EM, Inserra F, Ferder L, Fraga CG. Enalapril and captopril enhance glutathione-dependent antioxidant defenses in mouse tissues. Am J Physiol Regul Integr Comp Physiol 2000; 278: R572-7.

52. Raghavendra V, Chopra K, Kulkarni SK. Comparative studies on the memory-enhancing actions of captopril and losartan in mice using inhibitory shock avoidance paradigm. Neuropeptides 2001; 35: 65-9.

53. Mohamed DI, Khairy E, Tawfek SS, Habib EK, Fetouh MA Coenzyme Q10 attenuates lung and liver fibrosis via modulation of autophagy in methotrexate treated rat. Biomed Pharmacother 2019; 109: 892-901.

54. Bartesaghi R, Severi S, Guidi S. Effects of early environment on pyramidal neuron morphology in field CA1 of the guinea-pig. Neuroscience 2003; 116: 715-32.

55. Aboutaleb N, Shamsaei N, Rajabi H, et al. Protection of hippocampal ca1 neurons against ischemia/reperfusion injury by exercise preconditioning via modulation of Bax/Bcl-2 Ratio and prevention of Caspase-3 activation. Basic Clin Neurosci 2016; 7: 21-9.

56. Uttara B, Singh AV, Zamboni P, Mahajan RT. Oxidative stress and neurodegenerative diseases: a review of upstream and downstream antioxidant therapeutic options. Curr Neuropharmacol 2009; 7: 65-74.

57. Salganik RI. The benefits and hazards of antioxidants: controlling apoptosis and other protective mechanisms in cancer patients and the human population. J Am Coll Nutr 2001; 20: 464S-475S

58. da Silva MH, da Rosa EJ, de Carvalho NR, et al. Acute brain damage induced by acetaminophen in mice: effect of diphenyl diselenide on oxidative stress and mitochondrial dysfunction. Neurotox Res 2012; 21: 334-44.

59. Goldman SJ, Taylor R, Zhang Y, Jin S. Autophagy and the degradation of mitochondria. Mitochondrion 2010; 10 309-15.

60. Jin S. Autophagy, mitochondrial quality control, and oncogenesis. Autophagy 2006; 2: 80-4.

61. Gurer H, Neal R, Yang P, Oztezcan S, Ercal N. Captopril as an antioxidant in lead-exposed Fischer 344 rats. Hum Exp Toxicol 1999; 18: 27-32.

62. Trushina E. A shape shifting organelle: unusual mitochondrial phenotype determined with three-dimensional electron microscopy reconstruction. Neural Regen Res 2016; 11: 900-1.

63. Youle RJ, van der Bliek AM. Mitochondrial fission, fusion, and stress. Science 2012; 337: 1062-5.

64. Sofroniew MV, Vinters HV. Astrocytes: biology and pathology. Acta Neuropathol 2010; 119: 7-35.

65. Becerra-Calixto A, Cardona-Gomez GP. The role of astrocytes in neuroprotection after brain stroke: potential in cell therapy. Front Mol Neurosci 2017; 10: 88.

66. Ridet JL, Malhotra SK, Privat A, Gage FH. Reactive astrocytes: cellular and molecular cues to biological function. Trends Neurosci 1997; 20: 570-7.
67. Treuting PM, Dintzis SM, Montine KS. Comparative Anatomy and Histology: A Mouse, Rat, and Human Atlas, 2nd ed. Academic Press 2017.

68. Bylicky MA, Mueller GP, Day RM. Mechanisms of endogenous neuroprotective effects of astrocytes in brain injury. Oxid Med Cell Longev 2018; 2018: 6501031.

69. Dringen R, Gutterer JM, Hirrlinger J. Glutathione metabolism in brain metabolic interaction between astrocytes and neurons in the defense against reactive oxygen species. Eur J Biochem 2000; 267: 4912-16.

70. Won $\mathrm{R}$, Lee $\mathrm{KH}$, Lee $\mathrm{BH}$. Coenzyme $\mathrm{Q} 10$ protects neurons against neurotoxicity in hippocampal slice culture. Neuroreport 2011; 22: 721-6.

71. Bergamini C, Moruzzi N, Sblendido A, Lenaz G, Fato R. A water soluble $\mathrm{CoQ} 10$ formulation improves intracellular distribution and promotes mitochondrial respiration in cultured cells. PLoS One 2012; 7: e33712.

72. Matthews RT, Yang L, Browne S, Baik M, Beal MF. Coenzyme Q10 administration increases brain mitochondrial concentrations and exerts neuroprotective effects. Proc Natl Acad Sci U S A 1998; 95: 8892-7.

73. Beal MF. Coenzyme Q10 as a possible treatment for neurodegenerative diseases. Free Radic Res 2002; 36: 455-60.

74. Lee D, Kim KY, Shim MS, et al. Coenzyme Q10 ameliorates oxidative stress and prevents mitochondrial alteration in ischemic retinal injury. Apoptosis 2014; 19: 603-14.

75. Liang S, Ping Z, Ge J. Coenzyme Q10 regulates antioxidative stress and autophagy in acute myocardial ischemia-reperfusion injury. Oxid Med Cell Longev 2017; 2017: 9863181.

76. Sonsalla PK, Coleman C, Wong LY, et al. The angiotensin converting enzyme inhibitor captopril protects nigrostriatal dopamine neurons in animal models of parkinson ism. Exp Neurol 2013; 250: 376-83.

77. Karimani A, Mamashkhani Y, Jafari AM, Akbarabadi M, Heidarpour M. Captopril attenuates diazinon-induced oxidative stress: a subchronic study in rats. Iran J Med Sci 2018; 43: 514-22. 